\title{
Occurrence and Anti-Microbial Susceptibility Pattern of Extended Spectrum Beta-Lactamase Producing Enterobacteriaceae in Governmental Hospitals Wastewater in Addis Ababa, Ethiopia
}

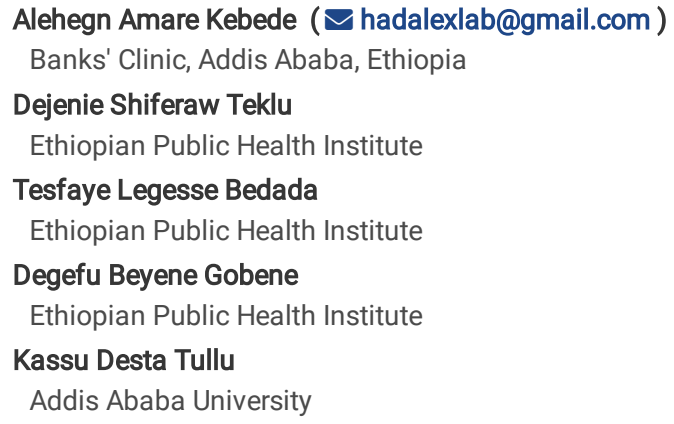




\section{Abstract \\ Background}

Worldwide, come out and dissemination of Extended-spectrum beta-lactamases (ESBLs) producing Enterobacteriaceae had been warning the efficacy of antibiotics to treat an infection. Hospital Wastewater might be a reservoir of such kind of resistant bacteria. The aim of this study was to get information about the occurrence of ESBLs producing and antibiotic susceptibility pattern of Enterobacteriaceae in hospital wastewater release from five governmental hospitals in Addis Ababa, Ethiopia.

\section{Methods}

A cross-sectional study was carried out from April 1 to May 31, 2020. Using a "Grab sampling technique" a total of 100 discrete wastewater samples were collected from each ward/unit wastewater collecting manholes of five governmental hospitals. For isolation and characterization of Enterobacteriaceae a loopful of a well-mixed sample was streaked on to MacConkey agar. Antimicrobial susceptibility testing was performed using the Kirby-Bauer disk diffusion method on Muller Hinton agar (MHA). All Enterobacteriaceae isolates were screened using cefotaxime and ceftazidime for ESBLs production as per CLSI guideline. The ESBLs suspicious were confirmed by a combination disk test (CDT). Data were entered and analyzed by using SPSS version-20.

\section{Result}

Of all Enterobacteriaceae the potential and confirmed ESBLs producer were 55.1\% (81/147) and $48.3 \%$ (71/147) respectively. The highest ratio of ESBLsproducer Enterobacteriaceae within sampling unit was observed in adult ward (66.7\%) and laundry unit effluent (58.8\%); in addition, the highest ESBL production was detected from E. coli (21.8\%), K. pneumoniae (4.8\%) and Citobacterspp. (4.8\%). The most elevated resistance level of ESBL producer was observed to cefotaxime (95.8\%) and amoxacilline/clavunalate (93\%). Furthermore, ESBL producing Enterobacteriaceae showed higher co-resistance rates to ciprofloxacine (74.6\%) and cotrimoxazole (73.2\%). The overall magnitude of MDR isolates were observed in 64\% (94/147) of tested Enterobacteriaceae.

\section{Conclusion}

In our study MDR and ESBLs producing Enterobacteriaceae was higher in hospital effluents. This indicates a potential risk of disseminating these resistance bacteria and resistant genes to the community if the hospital wastewater discharged without getting appropriate treatment.

\section{Introduction}

Wastewater is a used poor quality water that embrace waste liquid of domestic, agricultural, commercial sources, industries, and hospital sources (1). The effluent from hospital contain a lot of drug resistant pathogens, a larger form of chemicals, solvents, disinfectants and a lot of risky materials like pharmaceuticals and radionuclides than domestic sewerage (2). As a result of this hospital effluent contains antibiotic residues that are enough to kill susceptible bacteria and at the same time increases the number of resistant bacteria (3).

The presence of antibiotic resistant microorganism in effluent and sewage system line is a growing public health concern (4). Because one of the main ways of diffusion of pathogenic and/or antibiotic resistant microorganism is thru water, soil and air. This phenomenon results multi-drug resistant microorganisms have been revealed in different water sources including rivers, lakes, groundwater and drinking water (5-8). The discharge of resistant bacteria to the receiving aquatic environment will create public health impact through, carrying transmissible gene, by acting as a vector or reservoir of resistant gene $(9,10)$.

Bacteria, viruses, and parasites (including worms and protozoans) are the types of disease causing agent in wastewater that are dangerous to humans. Among the foremost encountered bacteria's in hospital wastewater are Enterobacteriaceae especially K. pneumoniae and E. coli (11). The members of the Enterobacteriaceae are Gram-negative bacilli, which are usually resident in the gastrointestinal tract. Instance of such organisms consisting of $E$. coli, $K$. pneumoniae, E. cloacae, Citrobacter freundii and Proteus mirabilis.

In patients hospitalized in intensive care units (ICUs), the Enterobacteriaceae holds for about one third of all cases of ICU-acquired pneumonia, one third of all cases of ICU-acquired urinary tract infection, and 10-15\% of ICU-acquired bloodstream infections (12). This kind of Enterobacteriaceae expelled as feces and other body fluids by in patient and out patients getting medical service then enter into hospital wastewater directly or through sewerage systems. Furthermore, they could transfer their resistant genes to another pathogenic and nonpathogenic bacteria found in wastewater via transferable plasmids. For this reason, assessing the occurrence of antimicrobial resistance and ESBLs producer Enterobacteriaceae in hospital wastewater is important in terms of strengthen infection prevention policy in hospital and avoiding discharge of hospital effluent into the community drainage without getting appropriate treatment.

Currently, the dominant antibiotics used for treating hospitalized patients infected by Gram-negative bacteria are the $\beta$-lactam antibiotics (because of their efficacy and less side effects) that inhibit transpeptidases taking part in bacterial cell wall synthesis. Sadly, these beta-lactam antibiotics will be deactivated by $\beta$-lactamase enzymes (13). A persistent exposure of bacterial strains to a multitude of beta-lactam antibiotics has evoked a dynamic, continuous production and mutation of beta lactamase in the bacteria resulting in the event of extended spectrum beta-lactamases (ESBLs) inflicting resistance to broad spectrum beta-lactam antibiotics (13-16). Hence, this contributes major increase in antibiotic resistance development specifically ESBLs.

The first ESBLs were isolated in the 1980's $(17,18)$. ESBLs are unremarkably outlined as beta-lactamases that confer resistance to the penicillins; first, second, and third generation cephalosporins and monobactams by hydrolysis of these antimicrobials. Additionally, these enzymes are pent-up by beta-lactamase

Page 2/18 
inhibitors such as clavulanic acid, sulbactam, and tazobactam. ESBLs are encoded on transferrable conjugative plasmids that facilitate widespread dissemination, not solely between the identical species of bacteria, but also across completely different species. Furthermore, these plasmids code for resistance to other classes of potent antimicrobial agents, in significantly, aminoglycosides and fluoroquinolones (19). ESBLs are isolated from a large type of Enterobacteriaceae (foremost in E. coli and K. pneumoniae), Pseudomonas aeruginosa and Capnocytophaga ochracea (20). The increasing prevalence of ESBL Entrobacteriaceae esp. K. pneumoniae and E. coli worldwide is a source of explicit concern. The hospital sewage or the low effectivity of hospital waste treatment plants may lead to the dissemination of ESBL-producing bacteria in to the community.

Our first objective was to generate information on the occurrence of antimicrobial resistance and ESBLs producer Enterobacteriaceae in Wastewater released from governmental hospital of Addis Ababa, Ethiopia. Currently, almost all hospitals in Addis Ababa neither had wastewater treatment plants (WWTPs) nor functional waste stabilization bond. Our second objective was to assess the frequency of the common Enterobacteriaceae and their antimicrobial susceptibility pattern. Enterobacteriaceae isolates were assessed for resistance pattern by using the Kirby-Bauer disk diffusion method, then confirmation of suspicious ESBL-producing isolate was verified by the combination disk method in line with 29th edition CLSI guideline (21).

\section{Methods Study Area}

A cross sectional study design was employed from April 1 to May 31, 2020 at the Ethiopian Public Health Institute (EPHI), Clinical Bacteriology and Mycology National Reference Laboratory in collaboration with Public Health microbiology in Addis Ababa, Ethiopia. Addis Ababa is the Capital City of the Federal Democratic Republic of Ethiopia, the diplomatic capital of Africa and the seat of different international and regional organizations. It hosts an estimated 3.238 million people, which is a $17 \%$ share of Ethiopia's total urban population. Presently, Addis Ababa is experiencing an annual growth rate of $3.8 \%$ and is speculated to reach 4.7 million residents by 2030 . The city covers a Landmass of 540 square kilo meters. The city is located at the heart of the country, at an altitude ranging from 2,100 meters at Akaki in the south to 3,000 meters at Entoto Hill in the North (22).

Within Addis Ababa city, there are 13 government hospitals (five federal, six under Addis Ababa health bureau, one owned by police force and one armed force hospital) distributed throughout 10 sub cities. Hospitals having 200 or more beds and large run of patients and those which provide 10 or more type of medical services were selected randomly which were five hospitals. The selected hospitals included: Tikur Ambesa Specialized Hospital (TASH) having 543 beds and providing 26 different medical service, St. Paul's Hospital Millennium Medical College(SPHMMC) with 337 beds and providing 25 different medical service, African leprosy and tuberculosis rehabilitation training center (ALERT) with 241 beds and providing 16 different medical service, Yekatit 12 hospital medical college (Y12HMC) with 210 beds and 19 different medical service and Menilik II referral hospital (MIIRH) with 203 beds and 15 different medical service (23).

\section{Sampling Frequency and Sampling Technique}

Hospitals that have higher bed and serving many patients were selected in ten sub- city of Addis Ababa. Based on these five governmental hospitals were included for this study. In each hospital, six sampling sites are employed to collect hospital effluent. These are a manhole used to collect a wastewater originate from adult ward, pediatric ward, labor ward, laboratory unit, laundry unit and a manhole of septic tank (which hold effluent from all source and we termed Mixed). In addition, one sampling unit viz. MDR TB ward was incorporated to collect hospital effluent only in alert hospital.

A total of 100 discrete (that is 50 at the morning and 50 at the afternoon) wastewater samples were collected from each ward/unit wastewater collecting manholes at the sampling site with four hour interval in the study period. This encompassed discrete effluent of 24 from each $\mathrm{Y} 12 \mathrm{HMC}$ and $\mathrm{MIIRH}, 20 \mathrm{from}$ each SPHMMC and ALERT, and 12 discrete effluents from TASH.

A "Grab sampling technique" was applied to collect the most representative samples according to guidelines of wastewater sampling techniques stated on EPA (24) and APHA (25). Discrete samples were collected in two rounds in each hospital for two months. The 1 st and 2 nd round samples were collected within 15 day interval. In each round, the discrete samples were collected two times a day with four hour interval from each sampling sites in each hospital in $150 \mathrm{ml}$ cleaned and sterile microbiological glass bottles. Here $150 \mathrm{ml}$ sterile glass container was used to collect $125 \mathrm{ml}$ wastewater samples.

Hospitals' effluent Samples were collected during their maximum activity period (usually 10:00 am- 2:30 pm) according to the method used by Nuñez and Moretton (10). In addition, samples were collected near the center of flow channel, at approximately 10-15 centimeter of the water depth, where the turbulence was at maximum and the possibility settling was minimized. Grazing (skimming) the water surface or slogging the bottle was avoided. The first sample was collected in the morning 10-10:30 AM whereas the second sample was collected at 2-230 PM from each sampling site. After taking the sample, the neck of the bottle was wiped with $95 \%$ alcohol then the sample bottle labelled with date, code number, and time and sampling site. All samples were collected manually and transported instantly to Ethiopia Public Health Institute (EPHI) food microbiology and clinical laboratories with cold chin $\left(4^{\circ} \mathrm{C}\right)$ for bacteriological analysis within six hours of collection. A pair of new, non-powdered, disposable glove, a suitable gown and eye google were used each time while, collecting samples to avoid personal contamination. In the same token, heavy duty glove was used to clean and pick up the cover of manholes at time of sample collection.

\section{Sampling Site}

In present study, there were different wards and units in the selected hospitals. Each ward/unit generated wastewater having different characteristics. So in order to locate in which sampling site the isolate found, the hospital effluents were collected at different manhole of each units/wards of hospital. The hospital effluents were collected at manhole of the adult ward, pediatric ward, delivery ward, laboratory unit, laundry service unit and at sampling site named MIXED (for this study purpose only). Here mixed sample indicates a hospital effluent in which its origin holding from different ward/unit and flow together

Page $3 / 18$ 
hence it was difficult to identify at which specific unit/ward it came. In addition, wastewater was collected from MDR TB ward in case of Alert hospital only. The sampling site manholes located just at the outlet of wastewater of each ward/unit before discharged in to receiving water/ collecting septic tank and at the side of each ward/unit building. The geographical position of the sampling site/ unit was obtained and documented.

\section{Data Collection Procedure}

The important information's were recorded using a pre-developed data collection form by asking the authorized body (about sampling unit/site, wastewater disinfection and disposal procedures), from record book/file (e.g. number of patients served during study period) and using google map application (for geographical location of the sample). After the sampling unit/site at each hospitals identified and its location as well as the source of wastewater, aseptically with care, the cover of each manholes are lifted up to collect the wastewater then covered immediately. The name of the hospital and sampling unit, time and round of collection, as well as its geographical location are recorded on pre-developed data collection format in addition to, labeling collecting bottle at time of sample collection. All of this information was collected by the principal investigator.

\section{LABORATORY ANALYSIS}

\section{Isolation and Characterization of Pure Cultures}

For isolation the bacteria, a loopful of a well-mixed sample suspension was inoculated using sterile inoculating loop on to MacConkey agar pate (Oxoid LTD, Basingstoke, Hampshire, England) and incubated aerobically at $37^{\circ} \mathrm{C}$ for $24-48$ hours.

After incubation for $24 \mathrm{hr}$. at $37^{\circ} \mathrm{C}$, bacterial colonies with distinct coloration and morphology were randomly picked up and sub cultured on to another MacConkey Agar plate for further purification. Then purified colonies with distinct presumptive colonies of each suspected bacterial species and fermentation on MacConkey agar are sub cultured on tryptic soya agar (TSA)/nutrient agar (Oxoid LTD, Basingstoke, Hampshire, England) depending on the availability of media for biochemical test.

For identification pure colony from non-selective nutrient agar/TSA was sub cultured and identified based on the following biochemical tests: oxidase, indole, urea, motility, Lysine decarboxylase, citrate utilization and triple sugar iron tests as per the standards of microbiology procedure including $E$. coli ATCC 25922 , as a control culture (26). Following purification and species identified, two -three purified colonies were preserved in Skimmy milk at $-80^{\circ} \mathrm{C}$ for further characterization, after each isolate was assigned a unique identification number.

\section{Screening isolates for ESBLs Producing}

Those Enterobacteriaceae that was resistant or reduced susceptibility to the screening indicator cephalosporin (cefotaxime and/or ceftazidime) was considered as suspicious of ESBLs production. In other word, isolates that showed an inhibition zone size of $\leq 27 \mathrm{~mm}$ for cefotaxime (30 $\mu$ g) and/or $\leq 22 \mathrm{~mm}$ for ceftazidime $(30 \mu \mathrm{g})$ were considered as suspicious ESBL producers and selected for confirmation for ESBLs production inline with 29 th edition clinical and laboratory standard institute (CLSI) guidelines (21).

\section{Confirmation of ESBLs Producing Enterobacteriaceae}

Confirmation of suspicious ESBL-producing isolate was verified by the combination disk method as delineated by the 29th edition CLSI guide line (21). The test was performed using two cephalosporin antibiotics: ceftazidime $(30 \mu \mathrm{g})$, and cefotaxime $(30 \mu \mathrm{g})$ alone and in combination with beta-lactam inhibitor ((ceftazidime- clavulanic acid $(30 / 10 \mu \mathrm{g})$, and cefotaxime-clavulanic acid $(30 / 10 \mu \mathrm{g})$ ) by dispensing on $0.5 \mathrm{McF}$ arland turbidity bacterial suspension inoculated Muller Hinton agar (MHA) plate (Oxoid LTD, Basingstoke, Hampshire, England) and then incubated overnight (18-24 hours) at $37^{\circ} \mathrm{C}$ as per 29 th edition $\mathrm{CLSI}$ guideline. ESBL production was considered positive when $\geq 5 \mathrm{~mm}$ increase in the zone diameter for the ceftazidime or cefotaxime tested in combination with clavulanic acid versus its zone when tested alone (21). E. coli ATCC 25922 was used as a negative control throughout the tests as a non-ESBL culture.

\section{Antimicrobial Susceptibility Testing}

Once the bacteria were isolated and identified from each sample collected, all Enterobacteriaceae isolates were assessed for non-susceptible pattern for 12 antibiotic agents by using the Kirby-Bauer disk diffusion method on MHA in line with 29th edition CLSI guideline (21). Bacterial inoculum was prepared by suspending the freshly grown bacteria in $4-5 \mathrm{ml}$ sterile normal saline and the turbidity was adjusted to that of a $0.5 \mathrm{McFarland}$ standard. Then a prepared bacterial inoculum suspension ( $0.5 \mathrm{McF}$ arland standards) was streaked on MHA using sterile swap applicator stick and antimicrobial discs were placed. The antibiotic discs used for this study were: trimethoprim/ sulphametoxazole (SXT, 1.25/3.75ug), ciprofloxacin (CPR, 5ug), tazobactam + piperacillin (TZP, 30ug),

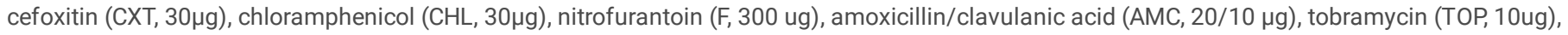
meropenem (MER, 10 $\mu$ g), cefotaxime (CTX, 30ug), cefepime (CFP, $30 \mu \mathrm{g})$, and ceftazidime (CAZ, $30 \mu \mathrm{g})$. The antibiotic discs used were the product of Abtek Biologicals Ltd, Liverpool, United Kingdom. Inhibition zones were measured using ruler and isolates were categorized as: resistance, intermediate and susceptible for each antimicrobial agent using the break point as set in line with 29th edition CLSI guidelines (21). The isolates were going to be considered as MDR when they were non- susceptible for three or more classes of antibiotics (27). E. coli ATCC 25922 and $P$. aeruginosa ATCC 27853 were used for quality control throughout the antimicrobial susceptibility tests as recommended by 29 th edition CLSI.

\section{Laboratory Data Quality Assurance}

Sample collection, handling, transportation and microbiological analysis and interpretation of results were carried out using standard operating procedures. Before the tangible procedure; reagents, antimicrobial disks, and media were checked for damage, storage problems and expiry date. Laboratory equipment's are appropriately cleaned and sterilized before use. Media's was prepared according to the respective manufacturer's instruction. Five percent of prepared 
media per batch was incubated overnight for sterility checkup. Quality control for new batch was performed using ATCC 25922 E. coli standard control to cross check the quality of antibiotics disks and culture media. For ESBLs confirmatory test E. coli ATCC 25922 (ESBLs negative) and K. pneumoniae ATCC 700603 (ESBLs positive) standard control strains are served at time of the procedure (21).

\section{Data analysis and interpretation method}

Data was entered and summarized using SPSS version 20 software (IBM Corporation, Armonk, NY, USA). Frequency and percentages of isolates, antibiotic susceptibility pattern of Enterobacteriaceae and ESBL producing Enterobacteriaceae were calculated. Tables and figures have been employed for data demonstration.

\section{Statistical Data Quality Assurance}

Before data entry, data from the data collection form was cross checked for its completeness and accuracy. Culture isolates and antibiotics susceptibility test results had been documented consciously ahead of entry to SPSS. Furthermore, data cleaning and double-data entry was implemented to assure quality of the data.

\section{Operational Definitions}

Multi-drug resistant (MDR): when a bacteria simultaneously non susceptible to at least three different classes of antimicrobial agents.

Non susceptible: are those bacteria which are resistant and intermediate for antibiotics susceptibility

Extended spectrum bata-lactamase producing Enterobacteriaceae: are bacteria that produce beta-lactamase enzymes to resist penicillins, first, second, and third generation cephalosporins and monobactams antibiotics by hydrolysis of these antimicrobials, but not cephamycins (e.g. cefoxitin) or carbapenems (meropenem).

Potential (Suspicious) ESBLs producing Enterobacteriaceae: are those bacteria which showed cefotaxime zone of inhibition $\leq 27 \mathrm{~mm}$ and ceftazidime zone of inhibition $\leq 22 \mathrm{~mm}$ ( viz. ESBLs screening positive)

Non-ESBLs suspicious/potential Enterobacteriaceae: are bacteria that are ESBLs screening negative

Non-ESBLs Enterobacteriaceae: are bacteria including Non-ESBLs suspicious/potential and confirmed ESBLs negative Enterobacteriaceae.

\section{Results}

\section{Distribution of Gram-negative Bacteria Isolates against Sampling Unit and Hospitals}

A total of 100 hospital effluent samples were collected and analyzed for the presence of Enterobacteriaceae family. Of these samples $87 \%$ were positive to one or more isolates. Meanwhile, 183 non-duplicate Gram-negative bacteria were picked from MacConkey agar, 80.3\% (147/183) belonging to Enterobacterial species. The remaining isolates included Pseudomonas spp. (2.2\%), Acinetobacter spp. (4.4\%), and other unidentified Gram-negative bacteria (13\%). Of 147 Enterobacteriaceae family isolates recovered the highest distributions were from; laboratory unit 32 (21.8\%) and mixed source effluent 30 (20.4\%); whereas, the least isolates were recovered from MDR TB ward effluent 7 (4.8\%), which was only collected from ALERT. In this study, the highest number of isolated bacteria, irrespective of total sample collected, were recovered from Y12HMC (34) and MIIRH (32); while, the least isolates were obtained from TASH (21) (Table 1).

\section{Frequency of Enterobacteriaceae Isolates}

Among all Enterobacteriaceae the most frequent isolates were E. coli (45.6\%), K. pneumoniae (10.2 \%) and E. cloacae (9.5\%) respectively. E. coli was predominantly isolated in laboratory effluent (22.4\%) followed by adult ward effluent (19.4\%). Likewise K. pneumoniae were mostly obtained from laboratory effluent (26.7\%). E. coli and K. pneumoniae were the most frequently isolate identified from pediatric ward $(47.4 \%, 10.5 \%)$, laboratory unit (46.9\%, $12.5 \%)$, and laundry unit $(58.8 \%, 17.6 \%)$ respectively. The common isolates detected from adult ward and mixed source effluents were $E$. coli $(61.9 \%, 33.3 \%)$ and Citrobacterspp $(19.5 \%, 13.3 \%)$ respectively. While, from labor ward E. coli (33.3\%) and K. oxytoca (14.3\%) and from MDR TB ward effluent $E$. coli (42.9\%) and M. morganii (28.6\%) were the frequently identified Enterobacteriaceae (Table 1). 
Table 1

Distribution of Enterobacteriaceae isolate against hospital, sampling unit, time and round of sample collection, at selected governmental hospitals wastewater, Addis Ababa, Ethiopia from April 1 to May 31, 2020

\begin{tabular}{|c|c|c|c|c|c|c|c|c|c|c|c|}
\hline \multicolumn{2}{|c|}{ Variables (n) } & \multicolumn{10}{|c|}{ Enterobacteriacae isolates $\mathrm{n}(\%)$} \\
\hline & & E. coli & $\begin{array}{l}\text { K. } \\
\text { pneumonia }\end{array}$ & $\begin{array}{l}\text { E. } \\
\text { cloacae }\end{array}$ & $\begin{array}{l}\text { Citrobacter } \\
\text { spp. }\end{array}$ & $\begin{array}{l}\text { K. } \\
\text { ozanae }\end{array}$ & $\begin{array}{l}\text { K. } \\
\text { rhinoscler }\end{array}$ & $\begin{array}{l}\text { K. } \\
\text { oxytoca }\end{array}$ & $\begin{array}{l}\text { M. } \\
\text { morganii }\end{array}$ & $\begin{array}{l}\text { Sallmonella } \\
\text { spp. }\end{array}$ & $\begin{array}{l}\text { Other } \\
\text { isolates* }\end{array}$ \\
\hline \multirow[t]{5}{*}{ Hospital } & TASH(21) & $\begin{array}{l}6 \\
(28.6)\end{array}$ & $2(9.5)$ & $4(19)$ & $1(4.8)$ & $3(14.3)$ & $3(14.3)$ & $1(4.8)$ & 0 & $1(4.8)$ & 0 \\
\hline & SPHMMC(32) & $\begin{array}{l}17 \\
(53.1)\end{array}$ & $3(9.4)$ & $2(6.3)$ & $1(3.1)$ & $2(6.3)$ & $1(3.1)$ & 0 & 1(3.1) & $1(3.1)$ & $4(12.5)$ \\
\hline & ALERT(28) & $\begin{array}{l}12 \\
(42.9)\end{array}$ & $3(10.7)$ & $2(7.1)$ & $4(14.3)$ & 0 & 0 & $2(7.1)$ & $2(7.1)$ & $1(3.6)$ & $2(7.1)$ \\
\hline & Y12HMC(34) & $\begin{array}{l}16 \\
(47.1)\end{array}$ & $3(8.8)$ & $3(8.8)$ & $4(11.8)$ & $1(2.9)$ & $3(8.8)$ & $2(5.9)$ & $1(2.9)$ & 0 & $1(2.9)$ \\
\hline & MIIRH(32) & $\begin{array}{l}16 \\
(50)\end{array}$ & $4(12.5)$ & $3(9.4)$ & $2(6.3)$ & $2(6.3)$ & $1(3.1)$ & $2(6.3)$ & 0 & 0 & $2(6.3)$ \\
\hline \multirow[t]{7}{*}{$\begin{array}{l}\text { Sampling } \\
\text { Unit }\end{array}$} & $\begin{array}{l}\text { Adult } \\
\text { ward(21) }\end{array}$ & $\begin{array}{l}13 \\
(61.9)\end{array}$ & $1(4.8)$ & $2(9.5)$ & $4(19)$ & 0 & 0 & 0 & 0 & $1(4.8)$ & 0 \\
\hline & $\begin{array}{l}\text { Pediatric } \\
\text { ward(19) }\end{array}$ & $\begin{array}{l}9 \\
(47.4)\end{array}$ & $2(10.5)$ & $2(10.5)$ & $1(5.3)$ & $2(10.5)$ & $1(5.3)$ & $1(5.3)$ & $1(5.3)$ & 0 & 0 \\
\hline & $\begin{array}{l}\text { Laboratory } \\
\text { unit(32) }\end{array}$ & $\begin{array}{l}15 \\
(46.9)\end{array}$ & $4(12.5)$ & $3(9.4)$ & 1(3.1) & 1(3.1) & $4(12.5)$ & 1(3.1) & 1(3.1) & 0 & $2(6.3)$ \\
\hline & $\begin{array}{l}\text { Laundry } \\
\text { unit(17) }\end{array}$ & $\begin{array}{l}10 \\
(58.8)\end{array}$ & $3(17.6)$ & $2(11.8)$ & 0 & 0 & 0 & 0 & 0 & 0 & $2(11.8)$ \\
\hline & $\begin{array}{l}\text { Labor } \\
\text { ward(21) }\end{array}$ & $\begin{array}{l}7 \\
(33.3)\end{array}$ & $2(9.5)$ & $2(9.5)$ & $1(4.8)$ & $1(4.8)$ & $2(9.5)$ & $3(14.3)$ & 0 & $1(4.8)$ & $2(9.5)$ \\
\hline & $\begin{array}{l}\text { Mixed } \\
\text { source(30) }\end{array}$ & $\begin{array}{l}10 \\
(33.3)\end{array}$ & $3(10)$ & $3(1)$ & $4(13.3)$ & $4(13.3)$ & 1(3.3) & $2(6.7)$ & 0 & $1(3.3)$ & $2(6.7)$ \\
\hline & $\begin{array}{l}\text { MDR TB } \\
\text { ward(7) }\end{array}$ & $\begin{array}{l}3 \\
(42.9)\end{array}$ & 0 & 0 & $1(14.3)$ & 0 & 0 & 0 & $2(28.6)$ & 0 & $1(14.3)$ \\
\hline \multirow[t]{2}{*}{$\begin{array}{l}\text { Time of } \\
\text { collection }\end{array}$} & Morning(71) & $\begin{array}{l}33 \\
(46.5)\end{array}$ & $10(14.1)$ & $7(9.9)$ & $4(5.6)$ & $1(1.4)$ & $4(5.6)$ & $3(4.2)$ & $3(4.2)$ & $3(4.2)$ & $3(4.2)$ \\
\hline & Aftemoon(76) & $\begin{array}{l}34 \\
(44.7)\end{array}$ & $5(6.6)$ & $7(9.2)$ & $8(10.5)$ & $7(9.2)$ & $4(5.3)$ & $4(5.3)$ & $1(1.3)$ & 0 & $6(7.9)$ \\
\hline \multirow[t]{2}{*}{$\begin{array}{l}\text { Round of } \\
\text { collection }\end{array}$} & First(80) & $\begin{array}{l}36 \\
(45)\end{array}$ & $7(8.8)$ & $8(10)$ & $6(7.5)$ & $5(6.3)$ & $5(6.3)$ & $5(6.3)$ & $2(2.5)$ & $2(2.5)$ & $4(5)$ \\
\hline & second(67) & $\begin{array}{l}31 \\
(46.3)\end{array}$ & 8 (11.9) & $6(9)$ & $6(9)$ & $3(4.5)$ & $3(4.5)$ & $2(3)$ & $2(3 \%)$ & $1(1.5)$ & $5(7.5)$ \\
\hline \multicolumn{2}{|c|}{ Total $(\mathrm{N}=147)$} & $\begin{array}{l}67 \\
(45.6)\end{array}$ & $15(10.2)$ & $14(9.5)$ & $12(8.2)$ & $8(5.4)$ & $8(5.4)$ & $7(4.8)$ & $4(2.7)$ & $3(2)$ & $9(6.1)$ \\
\hline
\end{tabular}

Note; *other isolates were Proteus spp, Shigella spp, Ent. aerogens, Edwardsella, and P. alkalifacience

TASH = Tikur Ambesa Specialized Hospital, SPHHMMC = St. Paul's Hospital Millennium Medical College, ALERT = All African leprosy and tuberculosis rehabilitation training center, $\mathrm{Y} 12 \mathrm{HMC}=$ Yekatit 12 hospital medical college, MIIRH = Menilik II referral hospital, MDR TB Ward = multidrug resistant tuberculosis war

\section{Antimicrobial Susceptibility pattern of Enterobacteriaceae}

The prevalence of antimicrobial non-susceptible pattern for Enterobacteriaceae isolated ranged from 8.2 to $77.6 \%$ in wastewater isolates, with most of the strains susceptible to meropenem (MER) and nitrofruntonine (F). Out of 147 Enterobacteriaceae strains tested, 125 (85\%) were found non-susceptible to at least one or more antibiotics tested (Table 2), with high non-susceptible to amoxicillin-clavulanic acid (77.6\%) followed by trimethoprim/sulfamethoxazole (57.8\%) and cefotaxime (53.7\%). Whereas, meropenem and nitrofurantoin had the lowest non-susceptible rate of $8.2 \%$ each (Table 1). Meanwhile, MDR strains, which were defined as resistant to at least three classes of antimicrobial agents, were mostly observed in the tested Enterobacteriaceae isolates by $64 \%(94 / 147)$ (Table 4). In the meantime, $15 \%$ of Enterobacteriaceae were susceptible for all tested antibiotics (Table 3).

A total of $62.7 \%$ E. coli, 83.3\% Citrobacterspp., $64.3 \%$ E. cloacae, and 53.3\% K. pneumoniae were identified as the predominant MDR isolates within species (Table 6). Of all Enterobacteriaceae isolates, $2.7 \%$ were non-susceptible to all antibiotics tested (12 drugs), $0.7 \%$ non-susceptible to ten drugs, and $12.9 \%$ were non-susceptible to seven drugs. Among Enterobacteriaceae isolates the most conspicuous MDR isolates, which were non-susceptible to 12 antibiotics tested detected in E. cloacae followed by in E. coli and Citrobacter spp. for 11 antibiotics (Table 3).

In this study the most first, second and third non-susceptible Enterobacteriaceae viz. E. coli, Citrobacter spp and E. cloace revealed the highest non-susceptible for amoxicillin-clavulanic acid of $85.1 \%, 75 \%$, and $85.7 \%$ respectively. However, the fourth most non-susceptible K. pneumoniae showed the highest level of 
non-susceptible against CTX (60\%) (Table 2).

Table 2

Antimicrobial non-susceptible pattern of Enterobacteriaceae identified from different sampling unit at selected governmental hospitals wastewater in Addis Ababa, Ethiopia from April 1 to May 31, 2020.

\begin{tabular}{|c|c|c|c|c|c|c|c|c|c|c|c|c|}
\hline \multirow{2}{*}{$\begin{array}{l}\text { Isolates } \\
\text { (number) }\end{array}$} & \multicolumn{12}{|c|}{ Tested Antibiotics Number $\mathrm{n}(\%)$ ) } \\
\hline & SXT & CPR & TZP & CXT & $\mathrm{CHL}$ & $\mathbf{F}$ & AMC & ТОВ & MER & CTX & CFP & CAZ \\
\hline E. coli (67) & $43(64.2)$ & $34(50.7)$ & $9(13.4)$ & $14(20.9)$ & $4(6)$ & 0 & $57(85.1)$ & 18(26.9) & $3(4.5)$ & $35(52.2)$ & $32(47.8)$ & $29(43.3)$ \\
\hline $\begin{array}{l}\text { K. rhinoscler } \\
\text { (8) }\end{array}$ & $4(50)$ & $4(50)$ & 0 & $2(25)$ & $3(37.5)$ & 0 & $7(87.5)$ & $4(50)$ & 0 & $4(50)$ & $4(50)$ & $4(50)$ \\
\hline $\begin{array}{l}\text { K. } \\
\text { pneumonia } \\
\text { spp (15) }\end{array}$ & $7(46.7)$ & $6(40)$ & 0 & 0 & $2(13.3)$ & 0 & $8(53.3)$ & $5(33.3)$ & 0 & $9(60)$ & $8(53.3)$ & $8(53.3)$ \\
\hline $\begin{array}{l}\text { Other } \\
\text { isolates *(9) }\end{array}$ & $5(55.6)$ & $7(77.8)$ & 3(33.3) & $2(22.2)$ & $2(22.2)$ & $2(22.2)$ & $7(77.8)$ & $2(22.2)$ & 1(11.1) & $4(44.4)$ & $4(44.4)$ & $4(44.4)$ \\
\hline $\begin{array}{l}\text { Citrobacter } \\
\text { spp.(12) }\end{array}$ & $8(66.7)$ & $7(58.3)$ & $1(8.3)$ & $7(58.3)$ & $5(41.7)$ & $2(16.7)$ & $9(75)$ & $2(16.7)$ & 2(16.7) & $6(50)$ & $5(41.7)$ & $6(50)$ \\
\hline $\begin{array}{l}\text { Sallmonella } \\
\text { spp.(3) }\end{array}$ & 1(33.3) & $2(66.7)$ & 0 & 0 & 1(33.3) & 0 & $3(100)$ & 1(33.3) & 1(33.3) & $2(66.7)$ & $2(66.7)$ & 2(66.7) \\
\hline $\begin{array}{l}\text { E. cloacae } \\
\text { (14) }\end{array}$ & $7(50)$ & $9(64.3)$ & $5(35.7)$ & $9(64.3)$ & $6(42.9)$ & $6(42.9)$ & $12(85.7)$ & $6(42.9)$ & $5(35.7)$ & $8(57.1)$ & $8(57.1)$ & $8(57.1)$ \\
\hline $\begin{array}{l}\text { K. oxytoca } \\
\text { (7) }\end{array}$ & $2(28.6)$ & $3(42.9)$ & 0 & $1(14.3)$ & $1(14.3)$ & 0 & $3(42.9)$ & $2(28.6)$ & 0 & $3(42.9)$ & $2(28.6)$ & $3(42.9)$ \\
\hline $\begin{array}{l}\text { K. ozanae } \\
\text { (8) }\end{array}$ & $5(62.5)$ & $2(25)$ & $1(12.5)$ & $4(50)$ & $2(25)$ & 0 & $5(62.5)$ & $3(37.5)$ & 0 & $5(62.5)$ & $5(62.5)$ & $4(50)$ \\
\hline $\begin{array}{l}\text { M.morganii } \\
\text { (4) }\end{array}$ & $3(75)$ & $3(75)$ & 0 & 0 & 0 & $2(50)$ & $3(75)$ & 0 & 0 & $3(75)$ & $3(75)$ & $3(75)$ \\
\hline $\begin{array}{l}\text { Total non- } \\
\text { susceptible }\end{array}$ & $85(57.8)$ & $77(52.4)$ & 19(12.9) & $39(26.5)$ & $26(17.7)$ & $12(8.2)$ & $114(77.6)$ & $43(29.3)$ & $12(8.2)$ & 79(53.7) & $73(49.7)$ & $71(48.3)$ \\
\hline
\end{tabular}

Note: SXT = Sulfamethoxazole-trimethoprim, CRP = Ciprofloxacin, TZP = Tazobactam/Piperacillin, CXT = Cefoxitin, CHL = Chloramphenicol, $\mathrm{F}=$ nitrofurantoin, $\mathrm{AMC}=$ Amoxicillin-Calvulanic acid, $\mathrm{TOB}=$ Tobramycine, $\mathrm{MER}=$ Meropenem, $\mathrm{CTX}=$ Cefotaxime, $\mathrm{CFP}=$ Cefepime, $\mathrm{CAZ}=\mathrm{Ceftazidime}$ * other isolates were Proteus spp, Shigella spp, Ent. aerogens, Edwardsella, and P. alkalifacience

Table 3

Level of antibiotic non-susceptible of Enterobacteriaceae identified from different sampling units of selected governmental hospitals wastewater, Addis Ababa, Ethiopia from April 1 to May 31, 2020.

\begin{tabular}{|c|c|c|c|c|c|c|c|c|c|c|c|c|c|}
\hline \multirow{2}{*}{$\begin{array}{l}\text { Isolates } \\
\text { (number) }\end{array}$} & \multicolumn{13}{|c|}{ Level of antibiotics non-susceptible ((number (\%)) } \\
\hline & Ro & $\mathbf{R} 1$ & $\mathbf{R} 2$ & R3 & R4 & R5 & R6 & R7 & R8 & R9 & R10 & R11 & R12 \\
\hline E. coli (67) & $6(9)$ & $8(11.9)$ & $11(16.4)$ & $8(11.9)$ & $4(6)$ & $7(10.4)$ & $8(11.9)$ & $5(7.5)$ & $4(6)$ & $4(6)$ & $1(1.5)$ & $1(1.5)$ & 0 \\
\hline $\begin{array}{l}\text { K. rhinoscler } \\
\text { (8) }\end{array}$ & $1(12.5)$ & 0 & $1(12.5)$ & $2(25)$ & 0 & 0 & $1(12.5)$ & $2(25)$ & $1(12.5)$ & 0 & 0 & 0 & 0 \\
\hline $\begin{array}{l}\text { K. pneumonia } \\
\text { (15) }\end{array}$ & $6(40)$ & $1(6.7)$ & 0 & 0 & 0 & $1(6.7)$ & $2(13.3)$ & $5(33.3)$ & 0 & 0 & 0 & 0 & 0 \\
\hline $\begin{array}{l}\text { Other isolates } \\
\text { (9) }\end{array}$ & 0 & 0 & $3(33.3)$ & $1(11.1)$ & $1(11.1)$ & $2(22.2)$ & 0 & 0 & 0 & $1(11.1)$ & 0 & $1(11.1)$ & 0 \\
\hline $\begin{array}{l}\text { Citrobacter } \\
\text { spp. (12) }\end{array}$ & 0 & 0 & $2(16.7)$ & $2(16.7)$ & $2(16.7)$ & $2(16.7)$ & $1(8.3)$ & $1(8.3)$ & $1(8.3)$ & 0 & 0 & $1(8.3)$ & 0 \\
\hline $\begin{array}{l}\text { Sallmonella } \\
\text { spp. (3) }\end{array}$ & 0 & $1(33.3)$ & 0 & 0 & 0 & 0 & 0 & $2(66.7)$ & 0 & 0 & 0 & 0 & 0 \\
\hline E. cloacae (14) & $2(14.3)$ & 0 & $3(21.4)$ & $1(7.1)$ & 0 & 0 & $1(7.1)$ & $1(7.1)$ & $1(7.1)$ & 0 & 0 & $1(7.1)$ & $4(28.6)$ \\
\hline K. oxytoca (7) & $4(57.1)$ & 0 & 0 & 0 & 0 & $1(14.3)$ & 0 & $1(14.3)$ & $1(14.3)$ & 0 & 0 & 0 & 0 \\
\hline K. ozanae (8) & $2(25)$ & 0 & $1(12.5)$ & 0 & 0 & $1(12.5)$ & $2(25)$ & 0 & $1(12.5)$ & $1(12.5)$ & 0 & 0 & 0 \\
\hline M.morganii (4) & $1(25)$ & 0 & 0 & 0 & 0 & 0 & $1(25)$ & $2(50)$ & 0 & 0 & 0 & 0 & 0 \\
\hline $\begin{array}{l}\text { TOTAL }(\mathrm{N}= \\
147)\end{array}$ & $22(15)$ & $\begin{array}{l}10 \\
(6.8)\end{array}$ & $\begin{array}{l}21 \\
(14.3)\end{array}$ & $\begin{array}{l}14 \\
(9.5)\end{array}$ & $7(4.8)$ & $\begin{array}{l}14 \\
(9.5)\end{array}$ & $\begin{array}{l}16 \\
(10.9)\end{array}$ & $\begin{array}{l}19 \\
(12.9)\end{array}$ & $9(6.1)$ & $6(4.1)$ & $\begin{array}{l}1 \\
(0.7)\end{array}$ & $4(2.7)$ & $4(2.7)$ \\
\hline
\end{tabular}

Note: R0: non-susceptible to no antibiotics, R1-12: non-susceptible to 1, 2, 3, 4, 5, 6-12 antibiotics used at time of study. 


\section{Magnitude of ESBLs Producing Enterobacteriaceae}

Of all Enterobacteriaceae 55.1\% (81/147) were suspected as potential ESBLs producing Enterobacteriaceae with screening method of cefotaxime zone of inhibition $\leq 27 \mathrm{~mm}$ and ceftazidime zone of inhibition $\leq 22 \mathrm{~mm}$ (Fig. 1). The overall magnitude of ESBLs producing Enterobacteriaceae was $48.3 \%$ (71/147) with the highest percentage found in E. coli, (21.8\%), K. pneumoniae (4.8\%) and Citobacter spp. (4.8\%); while, the lowest ratio observed in Salmonella spp. (1.4\%) (Figs. 1 and 2). The occurrence of ESBLs producers differ strongly within different species of Enterobacteriaceae. The highest within-species frequency of ESBLs production was recovered among M. morganii (75\%) pursued by Salmonella spp. (66.7\%) and K. ozanae (62.5\%) respectively. Meanwhile, least within-species ESBLs production was found in E. cloaca (21\%) (Table 5, and Fig. 2).

\section{Distribution of MDR and ESBLs producing Enterobacteriaceae against the variables}

From all hospital wastewater collected for this study purpose, the highest ratio of ESBLs- producer Enterobacteriaceae within sampling unit were detected in adult ward effluent (66.7\%) followed by laundry unit (58.8\%) and labor ward (47.6\%) respectively; whereas, the least proportion was recovered in pediatric ward $(36.9 \%)$ and laboratory unit effluent (40.6\%) (Table 5). Similarly with less difference, the elevated MDR isolates within sampling unit were identified in adult ward effluent (71.4\%) pursued by laundry unit (70.6\%) and laboratory unit effluents(62.5\%) correspondingly; while, the lowest ratio was found in pediatric ward (52.6\%) (Table 6). In MDR TB ward wastewater which was collected only in ALERT hospital, the proportions of ESBLs-producing and MDR Enterobacteriaceae within sampling unit were $57.1 \%$ and $85.7 \%$ respectively (Tables 5 and 6 ). Generally, of all MDR Enterobacteriaceae, $73.4 \%$ (69/94) were ESBLs producer, whereas only $26.6 \%$ (25/94) of them were non-ESBLs producer Enterobacteriaceae.

The highest occurrence of ESBL producing Enterobacteriaceae within hospital according to CDT identification method was found in wastewater from the ALERT (67.9\%) followed by TASH (52.4\%). Whereas, the least ESBL producing were occurred in MIIRH (31.2\%) (Table 5). In contrary, the elevated MDR isolates within hospital were observed in wastewater of TASH (76.2\%) and ALERT (71.4\%); while, the lowest ratio were identified in the same way as ESBL producer isolates in MIIRH (53.1\%) (Table 6). .

The magnitude of MDR and ESBL-producing Enterobacteriaceae obtained from all wastewater samples were higher at the afternoon than the morning wastewater collected. On the contrary, they were higher in the first round of effluent collection than the second (Tables 5 and 6 ). 
Table 4

Occurrence of MDR and ESBLs producing Enterobacteriaceae spp. Within hospital and sampling units as well as within MDR and ESBLs producer at selected hospitals wastewater, in Addis Ababa, Ethiopia from April 1 to May 31, 2020.

\begin{tabular}{|c|c|c|c|c|c|c|c|c|c|c|c|c|}
\hline \multicolumn{3}{|c|}{$\begin{array}{l}\text { Hospitals isolates n, Vs ESBLs } \\
\text { positive and MDR yes n (\%) }\end{array}$} & \multicolumn{7}{|c|}{ Sampling unit $\mathrm{n}(\%)$} & \multicolumn{2}{|c|}{$\begin{array}{l}\text { Wastewater collected } \\
\text { time }\end{array}$} & \multirow{2}{*}{$\begin{array}{l}\text { Waste } \\
\text { collect }\end{array}$} \\
\hline & & & $\begin{array}{l}\text { Adult } \\
\text { ward }\end{array}$ & $\begin{array}{l}\text { Pediatric } \\
\text { ward }\end{array}$ & Laboratory & Laundry & $\begin{array}{l}\text { Labor } \\
\text { ward }\end{array}$ & $\begin{array}{l}\text { Mixed } \\
\text { source }\end{array}$ & $\begin{array}{l}\text { MDR } \\
\text { TB } \\
\text { ward }\end{array}$ & Morning & Afternoon & \\
\hline \multirow[t]{15}{*}{$\begin{array}{l}\text { sample } \\
\text { collected } \\
\text { hospitals }\end{array}$} & $\begin{array}{l}\text { TASH, } \\
21\end{array}$ & $\begin{array}{l}\text { ESBLs } \\
\text { positive } \\
11(52.4)\end{array}$ & $1(9.1)$ & $2(18.2)$ & 2(18.2) & $1(9.1)$ & $2(18.2)$ & $3(27.3)$ & $\mathrm{NC}$ & $4(36.4)$ & $7(63.6)$ & $11(101$ \\
\hline & & MDR yes & $1(6.3)$ & $4(25)$ & $4(25)$ & $1(6.3)$ & $3(18.8)$ & $3(18.8)$ & $\mathrm{NC}$ & $8(50)$ & $8(50)$ & $16(101$ \\
\hline & & 16(31.2) & & & & & & & & & & \\
\hline & $\begin{array}{l}\text { SPHMMC, } \\
32\end{array}$ & $\begin{array}{l}\text { ESBLS } \\
\text { positive } \\
15(46.9)\end{array}$ & $2(13.3)$ & $3(20)$ & $2(13.3)$ & $4(26.7)$ & NA & $4(26.7)$ & $\mathrm{NC}$ & 7(46.7) & $8(53.3)$ & $4(26.7$ \\
\hline & & MDR yes & $4(18.2)$ & $3(13.6)$ & $4(18.2)$ & $5(22.7)$ & NA & $6(27.3)$ & $\mathrm{NC}$ & $11(50)$ & $11(50)$ & $9(40.9$ \\
\hline & & $22(68.8)$ & & & & & & & & & & \\
\hline & $\begin{array}{l}\text { ALERT, } \\
28\end{array}$ & $\begin{array}{l}\text { ESBLS } \\
\text { positive } \\
19(67.9)\end{array}$ & $4(21.1)$ & NA & $6(31.6)$ & $1(5.3)$ & $4(21.1)$ & NA & $4(21.1)$ & $9(47.7)$ & 10(52.6) & $12(63$. \\
\hline & & MDR yes & $3(15)$ & NA & $6(30)$ & $1(5)$ & $4(20)$ & NA & $6(30)$ & $10(50)$ & $10(50)$ & 11(55) \\
\hline & & $20(71.4)$ & & & & & & & & & & \\
\hline & $\begin{array}{l}\text { Y12HMC, } \\
34\end{array}$ & $\begin{array}{l}\text { ESBLs } \\
\text { positive } \\
16(47.1)\end{array}$ & $4(25)$ & $2(12.5)$ & $1(6.3)$ & 0 & $3(18.8)$ & $6(37.5)$ & $\mathrm{NC}$ & $8(50)$ & $8(50)$ & $9(56.3$ \\
\hline & & MDR yes & $4(21.1)$ & $3(15.8)$ & $1(5.3)$ & 0 & $4(21.1)$ & $7(36.8)$ & $\mathrm{NC}$ & $8(42.1)$ & $11(57.9)$ & $9(47.4$ \\
\hline & & 19(55.9) & & & & & & & & & & \\
\hline & $\begin{array}{l}\text { MIIRH, } \\
32\end{array}$ & $\begin{array}{l}\text { ESBLs } \\
\text { positive } \\
10(31.2)\end{array}$ & $3(30)$ & 0 & $2(20)$ & $4(40)$ & $1(10)$ & 0 & $\mathrm{NC}$ & $6(60)$ & $4(40)$ & $3(30)$ \\
\hline & & MDR yes & $3(17.6)$ & 0 & $5(29.4)$ & $5(29.4)$ & $2(11.8)$ & $2(11.8)$ & $\mathrm{NC}$ & $8(47.1)$ & $9(52.9)$ & $7(41.2$ \\
\hline & & 17(53.1) & & & & & & & & & & \\
\hline \multirow{4}{*}{$\begin{array}{l}\text { Total, } \\
\mathrm{N}=147\end{array}$} & & $\begin{array}{l}\text { ESBLs } \\
\text { positive }\end{array}$ & 14(19.7) & $7(9.9)$ & 13(18.3) & $10(14.1)$ & $10(14.1)$ & 13(18.3) & $4(5.6)$ & $34(47.9)$ & $37(52.1)$ & $39(54$ \\
\hline & & $\begin{array}{l}\mathrm{N}= \\
71(48.3 \%)\end{array}$ & & & & & & & & & & \\
\hline & & MDR yes & $15(16)$ & $10(10.6)$ & $20(21.3)$ & $12(12.8)$ & 13(13.8) & 18(19.1) & $6(6.4)$ & 45(47.9) & $49(52.1)$ & $52(53$ \\
\hline & & $\begin{array}{l}N= \\
94(64 \%)\end{array}$ & & & & & & & & & & \\
\hline
\end{tabular}

Note: TASH = Tikur Ambesa Specialized Hospital, SPHHMMC = St. Paul's Hospital Millennium Medical College, ALERT = All African leprosy and tuberculosis rehabilitation training center, $\mathrm{Y} 12 \mathrm{HMC}=$ Yekatit 12 hospital medical college, MIIRH = Menilik II referral hospital, MDR TB Ward = multidrug resistant tuberculosis ward, $\mathrm{NC}=$ sample not collected 
Table 5

Prevalence of ESBLs producing Enterobacteriaceae species within hospital, sampling unit, time and round of wastewater collection as well as with in species a producer at selected governmental hospitals wastewater, in Addis Ababa, Ethiopia from April 1 to May 31, 2020.

\begin{tabular}{|c|c|c|c|c|c|c|c|c|c|c|}
\hline \multicolumn{2}{|c|}{ ESBL POSETIVE, n (\%) } & \multicolumn{9}{|c|}{ Enterobactericae isolate identified n (\%) } \\
\hline & & E.coli & K.pneumonia & $\begin{array}{l}\text { Citrobacter } \\
\text { spp. }\end{array}$ & K.ozanae & K.rhinoscler & E.cloacae & K.oxytoca & M.morgani & $\begin{array}{l}\text { Sallmonella } \\
\text { spp }\end{array}$ \\
\hline \multirow{5}{*}{$\begin{array}{l}\text { wastewater } \\
\text { collected } \\
\text { hospitals }\end{array}$} & $\begin{array}{l}\text { TASH, } \\
11(52.4)\end{array}$ & 2(18.2) & $2(18.2)$ & $1(9.1)$ & $2(18.2)$ & $2(18.2)$ & 0 & $1(9.1)$ & 0 & $1(9.1)$ \\
\hline & $\begin{array}{l}\text { SPHMMC, } \\
15(46.9)\end{array}$ & $7(46.7)$ & $1(6.7)$ & 0 & 2(13.3) & 0 & 0 & 0 & $1(6.7)$ & $1(6.7)$ \\
\hline & $\begin{array}{l}\text { ALERT, } \\
19(67.9)\end{array}$ & $9(47.7)$ & $2(10.5)$ & $2(10.5)$ & 0 & 0 & $1(5.3)$ & $2(10.5)$ & $2(10.5)$ & 0 \\
\hline & $\begin{array}{l}\text { Y12HMC, } \\
16(47.1)\end{array}$ & $7(43.8)$ & $1(6.3)$ & $4(25)$ & $1(6.3)$ & $1(6.3)$ & $1(6.3)$ & 0 & 0 & 0 \\
\hline & $\begin{array}{l}\text { MIIRH, } \\
10(31.3)\end{array}$ & $7(70)$ & $1(10)$ & 0 & 0 & $1(10)$ & $1(10)$ & 0 & 0 & 0 \\
\hline \multirow{7}{*}{$\begin{array}{l}\text { wastewater } \\
\text { sampling } \\
\text { unit }\end{array}$} & $\begin{array}{l}\text { Adult ward, } \\
14(66.7)\end{array}$ & $9(64.3)$ & 0 & $3(21.4)$ & 0 & 0 & $2(14.3)$ & 0 & 0 & 0 \\
\hline & $\begin{array}{l}\text { Pediatric } \\
\text { ward, } \\
7(36.8)\end{array}$ & $3(42.9)$ & $1(14.3)$ & $1(14.3)$ & $2(28.6)$ & 0 & 0 & 0 & 0 & 0 \\
\hline & $\begin{array}{l}\text { Laboratory, } \\
13(40.6)\end{array}$ & $5(38.5)$ & $2(15.4)$ & $1(7.7)$ & $1(7.7)$ & $1(7.7)$ & 0 & $1(7.7)$ & $1(7.7)$ & \\
\hline & $\begin{array}{l}\text { Laundry, } \\
10(58.8)\end{array}$ & $7(70)$ & $2(20)$ & & & & 0 & & & \\
\hline & $\begin{array}{l}\text { Labor } \\
\text { ward, } \\
10(47.6)\end{array}$ & $2(20)$ & 0 & $1(10)$ & $1(10)$ & $2(20)$ & $1(10)$ & $1(10)$ & & $1(10)$ \\
\hline & $\begin{array}{l}\text { Mixed } \\
\text { source, } \\
13(43.3)\end{array}$ & $4(30.8)$ & $2(15.4)$ & $1(7.7)$ & $1(7.7)$ & $1(7.7)$ & 0 & $1(7.7)$ & & $1(7.7)$ \\
\hline & $\begin{array}{l}\text { MDR TB } \\
\text { ward, } \\
\mathbf{4 ( 5 7 . 1 )}\end{array}$ & $2(50)$ & & 0 & & & & & $2(50)$ & \\
\hline \multirow{2}{*}{$\begin{array}{l}\text { wastewater } \\
\text { collected } \\
\text { time }\end{array}$} & $\begin{array}{l}\text { Mornining, } \\
34(47.9)\end{array}$ & $15(44.1)$ & $5(14.7)$ & $2(5.9)$ & $1(2.9)$ & $2(5.9)$ & $1(2.9)$ & $1(2.9)$ & $2(5.9)$ & $2(5.9)$ \\
\hline & $\begin{array}{l}\text { Afternoon, } \\
37(48.7)\end{array}$ & $17(45.9)$ & $2(5.4)$ & $5(13.5)$ & $4(10.8)$ & $2(5.4)$ & $2(5.4)$ & $2(5.4)$ & $1(2.7)$ & \\
\hline \multirow{2}{*}{$\begin{array}{l}\text { wastewater } \\
\text { collected } \\
\text { round }\end{array}$} & $\begin{array}{l}\text { First, } \\
39(48.8)\end{array}$ & $17(43.6)$ & $3(7.7)$ & $3(7.7)$ & $3(7.7)$ & $3(7.7)$ & $1(2.6)$ & $3(7.7)$ & $2(5.1)$ & $2(5.1)$ \\
\hline & $\begin{array}{l}\text { Second, } \\
32(47.8)\end{array}$ & $15(46.9)$ & $4(12.5)$ & $4(12.5)$ & $2(6.3)$ & $1(3.1)$ & $2(6.3)$ & 0 & $1(3.1)$ & 0 \\
\hline \multirow[t]{4}{*}{ TOTAL } & $\begin{array}{l}\text { Total ESBL } \\
\text { Pos }\end{array}$ & $32(45.1)$ & $7(9.9)$ & $7(9.9)$ & $5(7)$ & $4(5.6)$ & $3(4.2)$ & $3(4.2)$ & $3(4.2)$ & $2(2.8)$ \\
\hline & $N=71$ & & & & & & & & & \\
\hline & $\begin{array}{l}\text { Total } \\
\text { Isolates }\end{array}$ & $67(47.8)$ & $15(46.7)$ & $12(58.3)$ & $8(62.5)$ & $8(50)$ & $14(21.4)$ & $7(42.9)$ & $4(75)$ & $3(66.7)$ \\
\hline & $N=147$ & & & & & & & & & \\
\hline
\end{tabular}

Note: TASH = Tikur Ambesa Specialized Hospital, SPHHMMC = St. Paul's Hospital Millennium Medical College, ALERT = All African leprosy and tuberculosis rehabilitation training center, $\mathrm{Y} 12 \mathrm{HMC}=$ Yekatit 12 hospital medical college, MIIRH = Menilik II referral hospital, MDR TB Ward = multidrug resistant tuberculosis ward 
Table 6

Prevalence of MDR Enterobacteriaceae species within hospital, sampling unit, time and round of wastewater collection as well as within species and MDR i selected governmental hospitals wastewater, in Addis Ababa, Ethiopia from April 1 to May 31, 2020.

\begin{tabular}{|c|c|c|c|c|c|c|c|c|c|c|}
\hline \multicolumn{2}{|c|}{ MDR YES, n (\%) } & \multicolumn{9}{|c|}{ Enterobacteriacae isolates recoverd from hospital effluents n(\%) } \\
\hline & & E.coli & $\begin{array}{l}\text { Citrobacter } \\
\text { spp. }\end{array}$ & E.cloacae & K.pneumonia & K.rhinoscler & K.ozanae & K.oxytoca & M.morganii & $\begin{array}{l}\text { Sallmonella } \\
\text { spp. }\end{array}$ \\
\hline \multirow{5}{*}{$\begin{array}{l}\text { wastewater } \\
\text { collected } \\
\text { hospitals }\end{array}$} & $\begin{array}{l}\text { TASH, } \\
16(76.2)\end{array}$ & $3(18.8)$ & $1(6.3)$ & $4(25)$ & $2(12.5)$ & $2(12.5)$ & $2(12.5)$ & $1(6.3)$ & & $1(6.3)$ \\
\hline & $\begin{array}{l}\text { SPHMMC, } \\
22(68.8)\end{array}$ & $10(45.5)$ & $1(4.5)$ & $1(4.5)$ & $2(9.1)$ & $1(4.5)$ & $2(9.1)$ & & $1(4.5)$ & $1(4.5)$ \\
\hline & $\begin{array}{l}\text { ALERT, } \\
20(71.4)\end{array}$ & $9(45)$ & $3(15)$ & $1(5)$ & $2(10)$ & & & $2(10)$ & $2(10)$ & 0 \\
\hline & $\begin{array}{l}\text { Y12HMC, } \\
19(55.9)\end{array}$ & $9(47.4)$ & $4(21.1)$ & $1(5.3)$ & $1(5.3)$ & $2(10.5)$ & $1(5.3)$ & 0 & 0 & \\
\hline & $\begin{array}{l}\text { MIIRH, } \\
17(53.1)\end{array}$ & $11(64.7)$ & $1(5.9)$ & 2(11.8) & $1(5.9)$ & $1(5.9)$ & 0 & 0 & & \\
\hline \multirow{5}{*}{$\begin{array}{l}\text { wastewater } \\
\text { sampling } \\
\text { unit }\end{array}$} & $\begin{array}{l}\text { Adult ward, } \\
15(71.4)\end{array}$ & $9(60)$ & $3(20)$ & 2(13.3) & $1(6.7)$ & & & & & 0 \\
\hline & $\begin{array}{l}\text { Pediatric } \\
\text { ward,10(52.6) }\end{array}$ & $5(50)$ & $1(10)$ & $1(10)$ & $1(10)$ & 0 & $2(20)$ & 0 & 0 & \\
\hline & $\begin{array}{l}\text { Laboratory, } \\
20(62.5)\end{array}$ & $7(35)$ & $1(5)$ & $2(10)$ & $2(10)$ & $3(15)$ & $1(5)$ & $1(5)$ & $1(5)$ & \\
\hline & $\begin{array}{l}\text { laundry, } \\
12(70.6)\end{array}$ & $8(66.7)$ & & $1(8.3)$ & $2(16.7)$ & & & & & \\
\hline & $\begin{array}{l}\text { Labor ward, } \\
13(61.9)\end{array}$ & $4(30.8)$ & $1(7.7)$ & $2(15.4)$ & 0 & $2(15.4)$ & $1(7.7)$ & $1(7.7)$ & & $1(7.7)$ \\
\hline \multirow{2}{*}{$\begin{array}{l}\text { wastewater } \\
\text { collected } \\
\text { Time }\end{array}$} & $\begin{array}{l}\text { Morning, } \\
45(63.4)\end{array}$ & $18(40)$ & $3(6.7)$ & $5(11.1)$ & 6(13.3) & $4(8.9)$ & $1(2.2)$ & $1(2.2)$ & $2(4.4)$ & $2(4.4)$ \\
\hline & $\begin{array}{l}\text { Afternoon, } \\
49(64.5)\end{array}$ & $24(49)$ & $7(14.3)$ & $4(8.2)$ & $2(4.1)$ & $2(4.1)$ & $4(8.2)$ & $2(4.1)$ & $1(2)$ & \\
\hline \multirow{2}{*}{$\begin{array}{l}\text { wastewater } \\
\text { collected } \\
\text { round }\end{array}$} & First, 52(65) & $22(42.3)$ & $5(9.6)$ & $5(9.6)$ & $4(7.7)$ & $3(5.8)$ & $3(5.8)$ & $3(5.8)$ & $2(3.8)$ & $2(3.8)$ \\
\hline & $\begin{array}{l}\text { Second, } \\
42(62.7)\end{array}$ & $20(47.6)$ & $5(11.9)$ & $4(9.5)$ & $4(9.5)$ & $3(7.1)$ & $2(4.8)$ & 0 & $1(2.4)$ & 0 \\
\hline \multirow[t]{2}{*}{ TOTAL } & $\begin{array}{l}\text { Total MDR N } \\
=94\end{array}$ & $42(44.7)$ & $10(10.6)$ & $9(9.6)$ & $8(8.5)$ & $5(6.4)$ & $5(5.3)$ & $3(3.2)$ & $3(3.2)$ & $2(2.5)$ \\
\hline & $\begin{array}{l}\text { Total Isolate } \\
N=147\end{array}$ & $67(62.7)$ & 12(83.3) & $14(64.3)$ & 15(53.3) & $8(62.5)$ & $8(62.5)$ & $7(42.9)$ & $4(75)$ & $3(66.7)$ \\
\hline
\end{tabular}

Note: TASH = Tikur Ambesa Specialized Hospital, SPHHMMC = St. Paul's Hospital Millennium Medical College, ALERT = All African leprosy and tuberculosis rehabilitation training center, $\mathrm{Y} 12 \mathrm{HMC}=$ Yekatit 12 hospital medical college, MIIRH = Menilik II referral hospital, MDR TB Ward = multidrug resistant tuberculosis ward

\section{Antibiotics Susceptibility pattern of ESBLs producing Enterobacteriaceae to potentially active drugs}

The antibiotic non-susceptible profile for ESBLs producing and non ESBLs producer Enterobacteriaceae are displayed in Fig. 3 below. The predominant ESBLs producer Enterobacteriaceae was found to be more than $85 \%$ non-susceptible, for antibiotics like cefotaxime (95.8\%), amoxacilline/clavunalate (93\%) and cefipeme (90.1\%). The most common co-resistance rates among the ESBL producing Enterobacteriaceae isolates to ciprofloxacin and trimthoprimesulfamethoxazole were $74.6 \%$ and $73.2 \%$ respectively. In addition, aminoglycoside and cephamycine such as tobramycine (40.8\%) and cefoxitine ( $29.6 \%$ ) showed reduced efficacy against the ESBL producing Enterobacteriaceae. However, the most active drugs for ESBLs producing isolates were meropenem and nitrofurantoin with susceptibility $94.4 \%$, and $88.7 \%$ correspondingly. Out of confirmed ESBLs producer Enterobacteriaceae the highest ESBLs production was observed among E. coli (45\%); while, the least ESBLs production was detected in salmonella spp. (2.2\%) (Table 5).

Non-ESBLs producers Enterobacteriaceae showed the highest non-susceptible level of $63.2 \%, 43.4 \%$ and $31.6 \%$ to amoxacilline/clavunalate, trimthoprimesulfamethoxazole and ciprofloxacin respectively. However, nitrofurantoin (7.9\%) and meropenem (10.5\%) showed the least non-susceptible to them.

\section{Discussion}




\section{Prevalence of Enterobacteriaceae isolates}

In the present study the most frequent Enterobacteriaceae isolates were E. coli (45.6\%), K. pneumoniae (10.2 \%) and E. cloacae (9.5\%). Our findings were comparable figure to other studies, in Addis Ababa; E. coli (32\%), K. pneumonia (15\%) and E. cloacae (6\%) (28), in South Eastern, Nigeria; E.coli (26.2\%) (29), in Luzhou City in Sichuan province, China; E. col i(56.5\%), K. pneumoniae (27.4\%) and Enterobacter spp.(8.1\%) (30), in Bangladish; E. coli (30.7\%), Klebsiella spp. (30.7\%) and Enterobacterspp. (25\%) (31). However, our finding was a little dissimilar to other studies conducted, in Northwest Ethiopia; from hospital environment Klebsiella spp. (29.2\%), E. coli (12.3\%) and Enterobacter spp. (3.1\%) (32), in Mekelle: from untreated hospital wastewater Klebsiella spp (25.9\%), and E. coli (21.2\%) (33), in Biratnagar, Nepal; from effluents of different hospitals sewage E. coli (34.7\%), Citrobacter (21.7\%), Enterobacter (21.7\%), and Klebsiella (13\%) (34). These variations might be due to sample type (inanimate object and swage of hospital), study period, sample size and type of pathogen infecting patients at time of sample collection.

\section{Antibiotics Non-susceptible pattern of Enterobacteriaceae isolates}

In the present study, the overall prevalence of antimicrobial non-susceptible pattern for Enterobacteriaceae isolated ranged from 8.2 to $77.6 \%$ in wastewater isolates, with most of the strains susceptible to meropenem (MER) and nitrofruntonine (F). This finding was in line with study conducted in Rio de Janeiro, Brazil, with 0 to $83 \%$ resistant range for Gram-negative isolates and most strains susceptible to meropenem (35).

In this study, out of 147 Enterobacteriaceae strains tested, 125 (85\%) were found non-susceptible to at least one or more antibiotics tested. Meanwhile, Enterobacteriaceae strains showed the highest non-susceptible to amoxicillin-clavulanic acid (77.6\%) followed by trimethoprim/sulfamethoxazole (57.8\%), cefotaxime (53.7\%), and ciprofloxacin (52.4\%).

This finding more or less correlates with other study conducted in China; trimethoprim/sulfamethoxazole (77.4\%), amoxicillin-clavulanic acid (66.1\%), and ciprofloxacin (61.3\%) (30) had relatively higher resistance. However, our finding disagrees with previous study conducted where lower non-susceptible proportion reported, in Northwest Ethiopia (Gondar):trimethoprim/sulfamethoxazole (29.8\%), cefotaxime (23.8\%), ciprofloxacin (10.6\%) (32), and in Bangiladish; ciprofloxcin (23\%) (31).

A study done in China showed high resistance of Enterobacteriaceae for cefotaxime (100\%), meropenem (51.6\%) and chloramphenicol (48.4\%) (30), contradicting the results presented herein where less non-susceptible observed for cefotaxime (53.7\%), chloramphenicol (17.7\%) and meropenem (8.2\%), again in Rio de Janeiro, Brazil: from the influent wastewater Gram-negative isolates showed resistant against cefotaxime (44\%),

trimethoprim/sulfamethoxazole (34\%), ciprofloxacine (17\%), and meropenem (3\%)(35), which were slightly deviate from current study except meropenem.

In present study, MDR strains were mostly observed in the tested Enterobacteriaceae isolates by $64 \%$. Almost similar MDR isolate results with ours were recorded in study carried out, in Northwest Ethiopia (Gondar) 81.5\% (from hospital environment) (32), in Mekelle: 61.5\% (from untreated hospital wastewater) (33), in Biratnagar, Nepal; 69.6\% (34), in China: 85.5\% (30). However, our report contradicted by the previous study conducted in South Eastern, Nigeria; (from three hospital effluents) where all the Enterobacteriaceae isolates recovered ( $E$. coli and Salmonella spp) were MDR although their patterns of resistance varied (29). In the same talked in this study, a total of $83.3 \%$ Citrobacter spp., $64.3 \%$ E. cloacae, $62.7 \%$ E. coli, and $53.3 \%$ K. pneumoniae isolates were identified as the predominant MDR. Our finding was concordant with other previous studies where the common MDR isolates were, in Addis Ababa; Citrobacter (100\%), E. cloacae (66.7\%) and E. coli (28.6\%) (28), in Biratnagar, Nepal; Enterobacter spp. (100\%), Citrobacter spp. (80\%), E. coli (62.5\%), Klebsiella spp (33.3\%) (34). However, our finding dissimilar with a study carried out in, China; (E. coli (91.4\%) and K. pneumoniae (94.1\%)) (30), Ibadan, Nigeria (E.coli (94.8\%)) (36), and Biratnagar, Nepal (Enterobacter spp. (100\%)) (34) where the highest MDR proportion for E. coli, K. pneumonia and Enterobacter spp. were indicated.

\section{Magnitude of Extended Spectrum B-lactamase producing Enterobacteriaceae}

In the present study, of all Enterobacteriaceae $55.1 \%$ were suspected as potential ESBLs producing and $87.7 \%$ of them were confirmed ESBLs producing isolates. A little comparable result was reported in Dubai, UAE by Khan MA. et a/ 2020: among all isolates from municipality wastewater $57.4 \%$ suspicious and 25.7\% confirmed ESBLs producer Enterobacteriaceae were reported (37), in Northern Italy: $45.4 \%$ beta-lactamases producing Enterobacteriaceae were recovered from WWTPs (38). The difference with our result might be due to the type of sample used (hospital Vs municipality wastewater), method used to confirm potential ESBLs producer (CDT Vs DDST) and sample size.

According to the present study, the overall magnitude of ESBLs producing Enterobacteriaceae were $48.3 \%$ which is almost in line with a study conducted in Rio de Janeiro, Brazil (35), and Nepal (34) with ESBLs producer isolates of 39\%, and $30.4 \%$ respectively. In contrast to the current study, other studies conducted in Ethiopia and other countries reported lower prevalence of ESBL producing Enterobacteriaceae, in Addis Ababa: $25 \%$ from hospital wastewater (28), in Northwest, Ethiopia: 14.8\% from hospital environment (39), and in Austria: $27.4 \%$ from activated sludge (40), were recovered (40). The difference in the prevalence of ESBLs producer in different studies from wastewater isolates might be due to difference in geographic areas, source of sample, period of study (ESBL rapidly changing over time), sample size, method of ESBL detection and an infection control system.

In the current study, the highest percentage of ESBLs producing Enterobacteriaceae was detected in E. coli (45.1\%), K. pneumoniae(9.9\%), and Citobacter spp (9.9\%) that is comparable results to study conducted in, Austria with E. coli (65.6\%), and K. pneumoniae (22.6\%) (40). and in Ibadan, Nigeria E.coli (29.3\%) (36). However, the highest ESBLs producer prevalence was documented in K. pneumoniae than E. coli or other Enterobacteriaceae spp. in other studies which contradict with the present study. Hence, the predominate ESBLs producing Enterobacteriaceae isolate in other studies were, in Addis Ababa; Citrobacter spp. (33.3\%), K.pneumonia (33.3\%), and E.coli (20\%) (28), in Northwest, Ethiopia, K. pneumoniae (42.10\%), and E. coli (35.09\%) (39), in Rio de Janeiro, Brazil: K. pneumonia (41.5\%), and E. coli (12.2\%) (35), and in Nepal; Enterobacterspp. (60\%), Citrobacterspp (40\%) and E. coli (25\%) (34). This variation is occurred 
because of the difference of, wastewater type (hospital Vs municipality), source of wastewater contaminant, the prevalence of microbes, geographical location and disease epidemiology.

The occurrence of ESBLs producers differ strongly within different species of Enterobacteriaceae. The highest within-species frequency of ESBLs production was recovered among M. morganii (75\%) pursued by Salmonella spp (66.7\%) and K. ozanae (62.5\%) respectively. Meanwhile, least within-species ESBLs production was found in E. cloaca (21\%).

The difference of the occurrence of ESBLs producer between within ESBLs producer and within species might be the variation of, the number of isolate recovered from the sample, and the isolate compared with (that is comparison within among ESBLs producer Enterobacteriaceae Vs within among each species).

\section{Distribution of MDR and ESBLs Producing Enterobacteriacae againist the Independent Variables}

In the present study, of all MDR Enterobacteriaceae, $73.4 \%$ were ESBLs producer, whereas only $26.6 \%$ of them were non-ESBLs producer Enterobacteriaceae. The magnitude of ESBLs producing and MDR Enterobacteriaceae in the wastewater were different in the five hospitals. The highest occurrence of ESBL producing Enterobacteriaceae within hospital according to CDT identification method were found in wastewater from the ALERT (67.9\%), followed by TASH $(52.4 \%)$ and Y12HMC (47.1\%) respectively, whereas; the least ESBL producing Enterobacteriaceae were detected in MIIRH (31.2\%). In contrary; the elevated MDR isolates within hospital were observed in wastewater of TASH (76.2\%) and ALERT (71.4\%), while; the lowest ratio was found in the same way as ESBL producer isolates in MIIRH (53.1\%). It is difficult to compare directly the occurrence of MDR and ESBLs producing Enterobacteriaceae in hospital effluent from one country to other because of the presence of difference in geographical zone, the epidemiology of disease (the severity and disease type), the number of patients served, the service provided in the hospital and wastewater disposal police from country to country. However, like our country there were different ESBLs producer occurrence within country hospital effluent, in Ibadan, Nigeria: more ESBLs producer was found in a privately-owned hospital (33.3\%) than a State Government-owned hospital (29.1\%) (36), in Europe: the elevated ESBLs producing Enterobacteriaceae was found in effluents from the Slovenian general hospital, followed by the Austrian private rehabilitation clinic and the Austrian private surgery clinic (41).

From all hospital wastewater collected for this study purpose, the highest ratio of ESBLs- producer Enterobacteriaceae within sampling unit was observed in adult ward effluent $(66.7 \%)$ followed by laundry unit (58.8\%) and labor ward effluents (47.6\%) respectively; whereas, the least proportion was recovered in pediatric ward (36.9\%) and laboratory unit effluent (40.6\%). Similarly with less difference; the elevated MDR isolates within sampling unit were identified in adult ward effluent (71.4\%) pursued by laundry unit (70.6\%) and laboratory unit effluents (62.5\%) correspondingly, while; the lowest ratio was found in pediatric ward effluent (52.6\%). In MDR TB ward wastewater which was collected only in ALERT hospital, the proportions of ESBLs-producing and MDR Enterobacteriaceae within sampling unit were $57.1 \%$ and $85.7 \%$ respectively. Almost all the preceding publication on antibiotic resistant profile of pathogenic microbes has been focused towards crude hospital wastewater rather than at each refined source of it. As a result, it was difficult to compare our result directly with other studies conducted from hospital wastewater, anyway a study conducted in hospital environment in Gondar, reported from inanimate object of medical ward, surgical ward and Gyn-obs ward ESBL producing Entrobacteriaceae of $52.6 \%, 10.5 \%$ and $5.3 \%$ respectively (39). The variation in ESBLsproducing and MDR Enterobacteriaceae proportion within sampling unit in the present study might probably be attributed to the difference in type of patients served, length of patient stay, type of medical service provided, infection prevention and control procedure in each department/unit.

In this study, the magnitude of MDR and ESBL-producing Enterobacteriaceae obtained from all wastewater samples were higher at the afternoon than the morning wastewater collected. At the afternoon effluent, the occurrence of MDR and ESBL producer isolates within time of wastewater collection were $64.5 \%$ and $52.1 \%$, whereas; at the morning effluent, were $59.2 \%$ and $47.9 \%$ respectively. This difference most probable happen because of the majority medical activity performed around the afternoon and outpatients number increase at the afternoon due to transportation and other reasons. On the other hand, MDR and ESBL-producing Enterobacteriaceae were higher in the first round of effluent collection than the second. In the first round, they were $77.6 \%$ and $48.8 \%$, while in the second round effluent collection, they were $62.7 \%$ and $40 \%$ correspondingly.

\section{Antibiotics susceptibility pattern of ESBLs producing Enterobacteriaceae}

In current study, the predominant ESBLs producer Enterobacteriaceae were found to be more than $85 \%$ non-susceptible to the antibiotic like cefotaxime (95.8\%), AMC (93\%), cefipeme (90.1\%), and ceftazidime (87.3\%). These were in close agreement with other study done in, Northwest Ethiopia; amoxicillin/clavulanic acid (100\%) and ceftazidime (100\%), Dubai, UAE; from municipality wastewater, cefotaxime (86\%) and ceftazidime (77\%) (37), Austria; amoxicillin/clavulanic acid (53.1\%) (40) had higher resistance for ESBLs producer Enterobacteriaceae.

In this study, the most common higher co-resistant rates among the ESBL producing Enterobacteriaceae isolates were $74.6 \%$ for ciprofloxacine and $73.2 \%$ for SXT. Whereas, aminoglycoside and cephamycine such as tobramycine (40.8\%) and cefoxitine (29.6\%) showed reduced efficacy against the ESBL producing Enterobacteriaceae. Our result was comparable with other studies taken place, in Northwest Ethiopia; ciprofloxacin (43.9\%), and trimethoprim/sulfamethoxazole (SXT) (64.9\%) (39), in Austria; ciprofloxacin (56.3\%), trimethoprim/sulfamethoxazole (50\%) and cefoxitin (25\%) (40).

In our work, nearly the most active drugs for ESBLs producing isolates observed are meropenem, nitrofurantoin, and piperacillin/tazobactam with susceptibility $94.4 \%, 88.7 \%$, and $87.3 \%$ and $74.6 \%$ respectively. The findings of this study nearly concordance with prior reports conducted in Austria; meropenem (100\%), and piperacillin/tazobactam (90.6\%) had good susceptibility level (40).

Non-ESBLs producers Enterobacteriaceae were $63.2 \%, 43.4 \%, 31.6 \%$ and $23.7 \%$ non-susceptible to amoxicillin/clavulanic acid, trimethoprim/sulfamethoxazole, ciprofloxacin and Cefoxitin respectively. However, nitrofurantoin, chloramphenicol and meropenem showed least non- 
susceptible with $7.9 \%, 10.5$ and $10.5 \%$ respectively. We observed in the present study ESBLs producer isolates were more resistant to the tested antibiotics than non-ESBLs producer Enterobacteriaceae. This difference might be from the resistant gene on ESBLs producer also contributed the isolate to develop resistance to other antibiotic too.

\section{Strength of the study}

Our study tried to collect the wastewater, at their utmost source, which enables to take preventive measure and at large sample size (in relative to previous study) to be representative. This study conducted at different governmental higher hospitals to display the extent of distribution of MDR and ESBLs producing Enterobacteriaceae in each hospital sampling units/sites.

\section{Limitation of the study}

Wastewater samples were taken over a short period of time and may not depict seasonal variation of isolates and it might be below the expected number. In this study only wastewater was used, hence it was unable to differentiate the source of non-susceptible bacteria either it was from clinical isolates or sewage system.

ESBL detection was only performed phenotypically using CDT method, it was better to include genotypic method of detection.

Some source of hospital wastewaters were not incorporated in the study. So to generalize the distribution of MDR and ESBLs producing Entrobacteriaceae in hospital wastewater, it was better assessing all source of wastewater in selected hospitals.

The study did not include private hospitals wastewater found in Addis Ababa and wastewater of WWTP of the city.

\section{Conclusion And Recommendation}

Nearly $65 \%$ MDR and $50 \%$ ESBLs producing Enterobacteriaceae was present in the hospital wastewater in this study, is a warning threat to such infection via contamination of food and water from rivers and urbane drainage which will be polluted through untreated hospital wastewater. The most common ESBLproducing Enterobacteriaceae were E. coli, K. pneumoniae and Citobacter spp. A higher level of non-susceptible to different classes of antibiotics was identified among ESBL producers relative to non-ESBL producers. ESBL-producing isolates showed the highest non-susceptible levels to cefotaxime, amoxacilline/clavunalate and cefipeme. These indicate inappropriate handling and disposal of the hospital effluent without getting the right treatment into the receiving community drainage may affect public health by contaminating the water bodies, soil and the environment, hence accelerate spreading of microorganisms and resistant genes into community. Consistently prevention strategies are needed to go beyond the stretch of these bacteria in the community and environment to inhibit the transmission of the antibiotic resistant to another enteric pathogenic bacterium. We recommended a highly committed hygienic treatment of effluent (implementation of final disinfection procedure to minimize the microbial burden) at each respected wards and units of the health institution.

\section{Abbreviations}

ALERT: All African leprosy and tuberculosis rehabilitation training center, AMR: Anti-Microbial Resistance, APHA: American Public Health Association, ATCC: American Type Culture Collection, CLSI: Clinical and Laboratory Standard Institute, EPA: Environment Protection Authority, EPHI: Ethiopian Public Health Association, ESBL: Extended Spectrum Beta-Lactamase, MDR TB Ward: Multidrug Resistant Tuberculosis ward, MDR: Multi-drug Resistance, MIIRH: Menilik II referral hospital, SPHHMMC: St. Paul's Hospital Millennium Medical College, SPSS: Statistical Package for the Social Sciences, TASH: Tikur Ambesa Specialized Hospital, WWTP: Wastewater Treatment Plant, Y12HMC:Yekatit 12 hospital medical college,

\section{Declarations}

\section{Ethics approval}

The study proposal was reviewed and verified by the department of research and ethics review committee of the medical laboratory sciences, College of Health Sciences; Addis Ababa University (Ref. №. MLS/10/19). Approval and permission letter was obtained from Addis Ababa health bureau and the respective hospital in which the hospital wastewater and data were collected by their authorized bodies.

\section{Consent for publication}

Not applicable

\section{Availability of data and material}

The current study data sets used for analysis can be obtained from the corresponding author through email (hadalexlab@gmail.com) on reasonable request.

\section{Competing interests}

The authors declare that they have no competing interests. 


\section{Funding}

Antibiotics disks and media were supplied by EPHI. However, the other costs were covered by the principal investigator. The supplier body had no influence on study design, data collection, analysis and interpretation of data and writing the manuscript.

\section{Authors' contributions}

AA: Conceived, designed, analyzed and interpreted the research; and also wrote the manuscript. KD: Supervised the study through their critical review of the research and the manuscript write up. DS, TL, DB: Participated in the technical laboratory works: All authors read and approved the final manuscript.

\section{Acknowledgment}

We are very grateful to thank EPHI and all microbiology laboratory staffs for giving us permission to conduct this research using the institution reusable equipment and by providing reagents and supplies from them in clinical bacteriology laboratories in collaboration with public health microbiology. Our sincere gratitude and thanks also goes to the five hospital medical director, infection prevention and control unit, and building repair and maintenance unit focal person for their loyal cooperative permission to conduct this research in their health institution as well as for their unreserved help without being bored off to show/indicate the sampling unit and its source accurately. Lastly but not lest we would like to give heart full thanks to Mr. Adane Amera for him close moral support, good and friendly approach advice, and follow up throughout this paper preparation.

\section{References}

1. Mahvi A, Rajabizadeh A, Yousefi N, Hosseini H, Ahmadian M. Survey wastewater treatment condition and effluent quality of Kerman province hospitals. World Applied Sciences Journal. 2009;7(12):1521-5.

2. Sharma P, Mathur N, Singh A, Bhatnagar P, Atri R, Sogani M. Efficiency analysis of a hospital effluent treatment plant in reducing genotoxicity and cytotoxicity of hospital wastewaters. Intl J of Adv Biotec Res. 2014;5(3):371-80.

3. Prasad V, Baliyan S, Sibi G. Prevalence of Antibiotic Resistance among Bacterial Isolates from Hospital Environments and Effluents. J Bacteriol Mycol. 2018;5(7):1082.

4. Reinthaler F, Posch J, Feierl G, Wüst G, Haas D, Ruckenbauer G, et al. Antibiotic resistance of E. coli in sewage and sludge. Water Res. 2003;37(8):168590.

5. Hamelin K, Bruant G, El-Shaarawi A, Hill S, Edge TA, Fairbrother J, et al. Occurrence of virulence and antimicrobial resistance genes in Escherichia coli isolates from different aquatic ecosystems within the St. Clair River and Detroit River areas. Appl Environ Microbiol. 2007;73(2):477-84.

6. Baquero F, Martínez J-L, Cantón R. Antibiotics and antibiotic resistance in water environments. Curr Opin Biotechnol. 2008;19(3):260-5.

7. Marti E, Jofre J, Balcazar JL. Prevalence of antibiotic resistance genes and bacterial community composition in a river influenced by a wastewater treatment plant. PLoS One. 2013;8(10):e78906.

8. Ramirez Castillo FY, Avelar González FJ, Garneau P, Marquez Diaz F, Guerrero Barrera AL, Harel J. Presence of multi-drug resistant pathogenic Escherichia coli in the San Pedro River located in the State of Aguascalientes, Mexico. Frontiers in microbiology. 2013;4:147.

9. Keen PL, Patrick DM. Tracking change: a look at the ecological footprint of antibiotics and antimicrobial resistance. Antibiotics. 2013;2(2):191-205.

10. Nunez L, Moretton J. Disinfectant-resistant bacteria in Buenos Aires city hospital wastewater. Brazilian Journal of Microbiology. 2007;38(4):644-8.

11. Oyeleke S, Istifanus N, Manga S. The effects of hospital solid waste on the receiving environment. International Journal of Integrative Biology. 2008;3(3):191-5.

12. Richards MJ, Edwards JR, Culver DH, Gaynes RP, System NNIS. Nosocomial infections in combined medical-surgical intensive care units in the United States. Infection Control Hospital Epidemiology. 2000;21(8):510-5.

13. Paterson DL, Bonomo RA. Extended-spectrum $\beta$-lactamases: a clinical update. Clin Microbiol Rev. 2005;18(4):657-86.

14. Chitnis V, Chitnis S, Vaidya K, Ravikant S, Patil S, Chitnis D. Bacterial population changes in hospital effluent treatment plant in central India. Water Res. 2004;38(2):441-7.

15. Pitout JD, Laupland KB. Extended-spectrum $\beta$-lactamase-producing Enterobacteriaceae: an emerging public-health concern. The Lancet infectious diseases. 2008;8(3):159-66.

16. Canton R, Novais A, Valverde A, Machado E, Peixe L, Baquero F, et al. Prevalence and spread of extended-spectrum $\beta$-lactamase-producing Enterobacteriaceae in Europe. Clinical Microbiology infection. 2008;14:144-53.

17. Brun-Buisson C, Philippon A, Ansquer M, Legrand P, Montravers F, Duval J. Transferable enzymatic resistance to third-generation cephalosporins during nosocomial outbreak of multiresistant Klebsiella pneumoniae. The lancet. 1987;330(8554):302-6.

18. Sirot D, Sirot J, Labia R, Morand A, Courvalin P, Darfeuille-Michaud A, et al. Transferable resistance to third-generation cephalosporins in clinical isolates of Klebsiella pneumoniae: identification of CTX-1, a novel $\beta$-lactamase. J Antimicrob Chemother. 1987;20(3):323-34.

19. Mahomed S, Coovadia YM. Faecal carriage of Extended Spectrum Beta-lactamase producing Escherichia coli and Klebsiella Pneumoniae in children from the community of Kwadedangendlale, KwaZulu-Natal, South Africa. International Journal of Infection Control. 2015;11(3).

20. Katsanis GP, Spargo J, Ferraro MJ, Sutton L, Jacoby GA. Detection of Klebsiella pneumoniae and Escherichia coli strains producing extended-spectrum beta-lactamases. J Clin Microbiol. 1994;32(3):691-6.

21. CLSI. Performance standards for antimicrobial susceptibility testing. CLSI supplement M100. ed. Wayne: Clinical and Laboratory Standards Institute; 2019. p. 29. 
22. Spaliviero M, Cheru F. The State of Addis Ababa 2017: the Addis Ababa we want. 2017.

23. FDRE MoH. National Medical Services Directory, version II. Addis Ababa: CDC, Tulan University; 2015. 1-189 p.

24. Duncan D, Harvey F, Walker MEPA, Guidelines. Regulatory Monitoring and Testing Water and Wastewater Sampling. Adelaide: Environment Protection Authorithy; 2007.

25. Association APH, Association AWW, Federation WPC, Federation WE. Standard methods for the examination of water and wastewater. American Public Health Association.; 1915.

26. Cheesbrough M. Manual of medical microbiology. Low price ed. Britain: Oxford press; 2000.

27. Magiorakos AP, Srinivasan A, Carey R, Carmeli Y, Falagas M, Giske C, et al. Multidrug-resistant, extensively drug-resistant and pandrug-resistant bacteria: an international expert proposal for interim standard definitions for acquired resistance. Clinical microbiology infection. 2012;18(3):268-81.

28. Tesfaye H, Alemayehu H, Desta AF, Eguale T. Antimicrobial susceptibility profile of selected Enterobacteriaceae in wastewater samples from health facilities, abattoir, downstream rivers and a WWTP in Addis Ababa, Ethiopia. Antimicrobial Resistance Infection Control. 2019;8(1):1-11.

29. Onuoha S. Isolation and Characterization of Multi-drug Resistant Bacterial Pathogens from Hospital Effluents, South Eastern, Nigeria. World Appl Sci J. 2017;35:82-7.

30. Zhang L, Ma X, Luo L, Hu N, Duan J, Tang Z, et al. The prevalence and characterization of extended-spectrum $\beta$-lactamase-and carbapenemase-producing bacteria from hospital sewage, treated effluents and receiving rivers. Int J Environ Res Public Health. 2020;17(4):1183.

31. Siddiqui MK, Khatoon N, Roy PC. Untreated liquid hospital waste: potential source of multidrug resistant bacteria. Bangladesh Journal of Microbiology. 2015:21-4.

32. Moges F, Endris M, Belyhun Y, Worku W. Isolation and characterization of multiple drug resistance bacterial pathogens from waste water in hospital and non-hospital environments, Northwest Ethiopia. BMC Res Notes. 2014;7(1):1-6.

33. Asfaw T, Negash L, Kahsay A, Weldu Y. Antibiotic resistant bacteria from treated and untreated hospital wastewater at Ayder Referral Hospital, Mekelle, North Ethiopia. Advances in Microbiology. 2017;7(12):871-86.

34. Mahato S, Mahato A, Adhikari P. Extended-Spectrum Beta-Lactamase-Producing Enterobacteriaceae in Effluents of Different Hospitals Sewage in Biratnagar, Nepal. International Journal of Environment. 2019;8(3):53-67.

35. Chagas TPG, Seki L, Cury J, Oliveira J, Dávila A, Silva D, et al. Multiresistance, beta-lactamase-encoding genes and bacterial diversity in hospital wastewater in Rio de Janeiro, Brazil. J Appl Microbiol. 2011;111(3):572-81.

36. Adekanmbi AO, Adeyemi AO, Olajide OM. Occurrence of multidrug resistant and extended spectrum $\beta$-lactamase (ESBL)-producing Escherichia coli in wastewater of two healthcare facilities in Ibadan, Nigeria. 26: World News of Natural Sciences An International Sientific Journal; 2019.

37. Khan MA, Thurgood NE, Faheem SM, Rais N, Ansari MZ, Kaleem SM, et al. Occurrence of Extended Spectrum Beta-Lactamase Gram-Negative Bacteria from Non-Clinical Sources in Dubai, United Arab Emirates. Water. 2020;12(9):2562.

38. Caltagirone M, Nucleo E, Spalla M, Zara F, Novazzi F, Marchetti VM, et al. Occurrence of extended spectrum $\beta$-lactamases, KPC-type, and MCR-1.2producing Enterobacteriaceae from wells, river water, and wastewater treatment plants in Oltrepò Pavese area, Northern Italy. Frontiers in microbiology. 2017;8:2232

39. Engda T, Moges F, Gelaw A, Eshete S, Mekonnen F. Prevalence and antimicrobial susceptibility patterns of extended spectrum beta-lactamase producing Entrobacteriaceae in the University of Gondar Referral Hospital environments, northwest Ethiopia. BMC Res Notes. 2018;11(1):1-7.

40. Galler H, Feierl G, Petternel C, Reinthaler FF, Haas D, Habib J, et al. Multiresistant bacteria isolated from activated sludge in Austria. Int J Environ Res Public Health. 2018;15(3):479.

41. Rozman U, Duh D, Cimerman M, Turk S. Hospital wastewater effluent: Hot spot for antibiotic resistant bacteria. Journal of Water Sanitation Hygiene for Development. 2020;10(2):171-8.

\section{Figures}




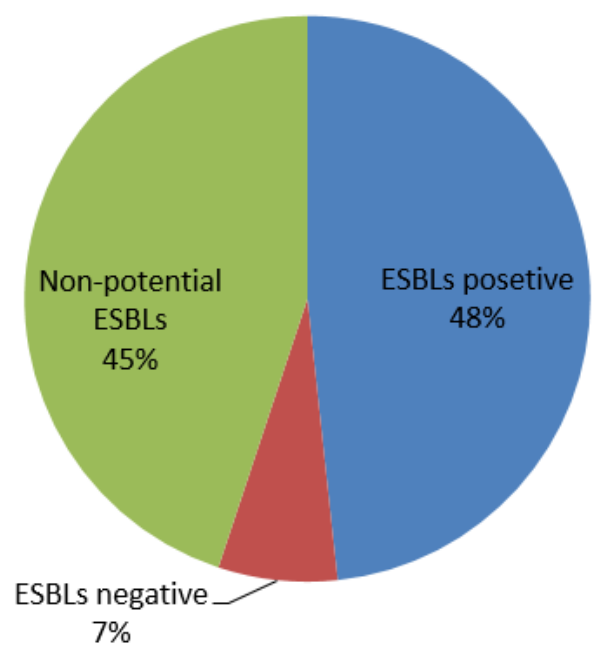

Figure 1

The percentage of ESBLs positive, ESBLs negative and non-potential ESBLs Enterobacteriaceae at selected governmental hospitals wastewater, Addis Ababa, Ethiopia, April 1 to May 31, 2020.

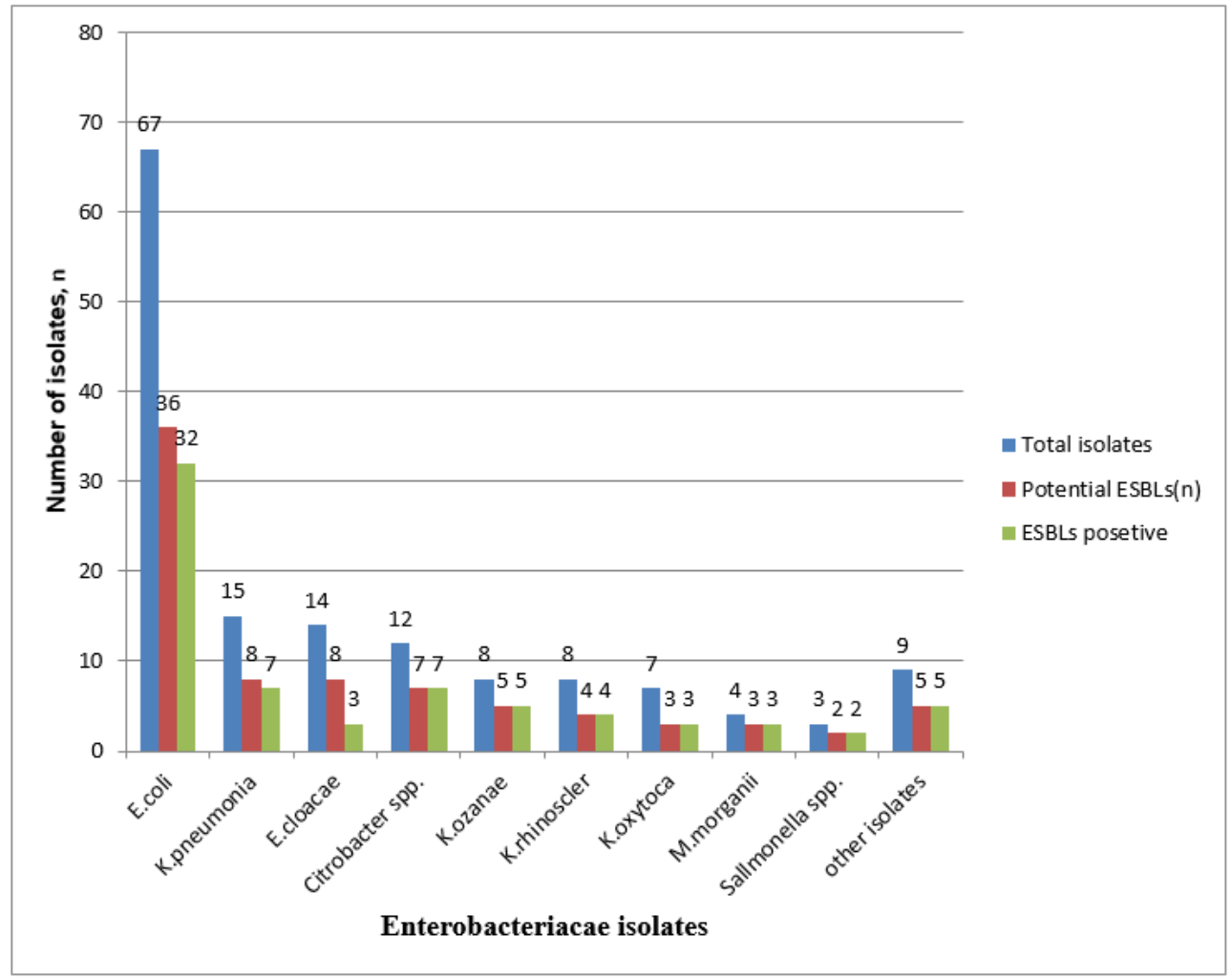

\section{Figure 2}

Frequency of total, potential and ESBLs producing Enterobacteriaceae species from different sampling units at selected governmental hospitals wastewater, in Addis Ababa, Ethiopia from April 1 to May 31, 2020. 


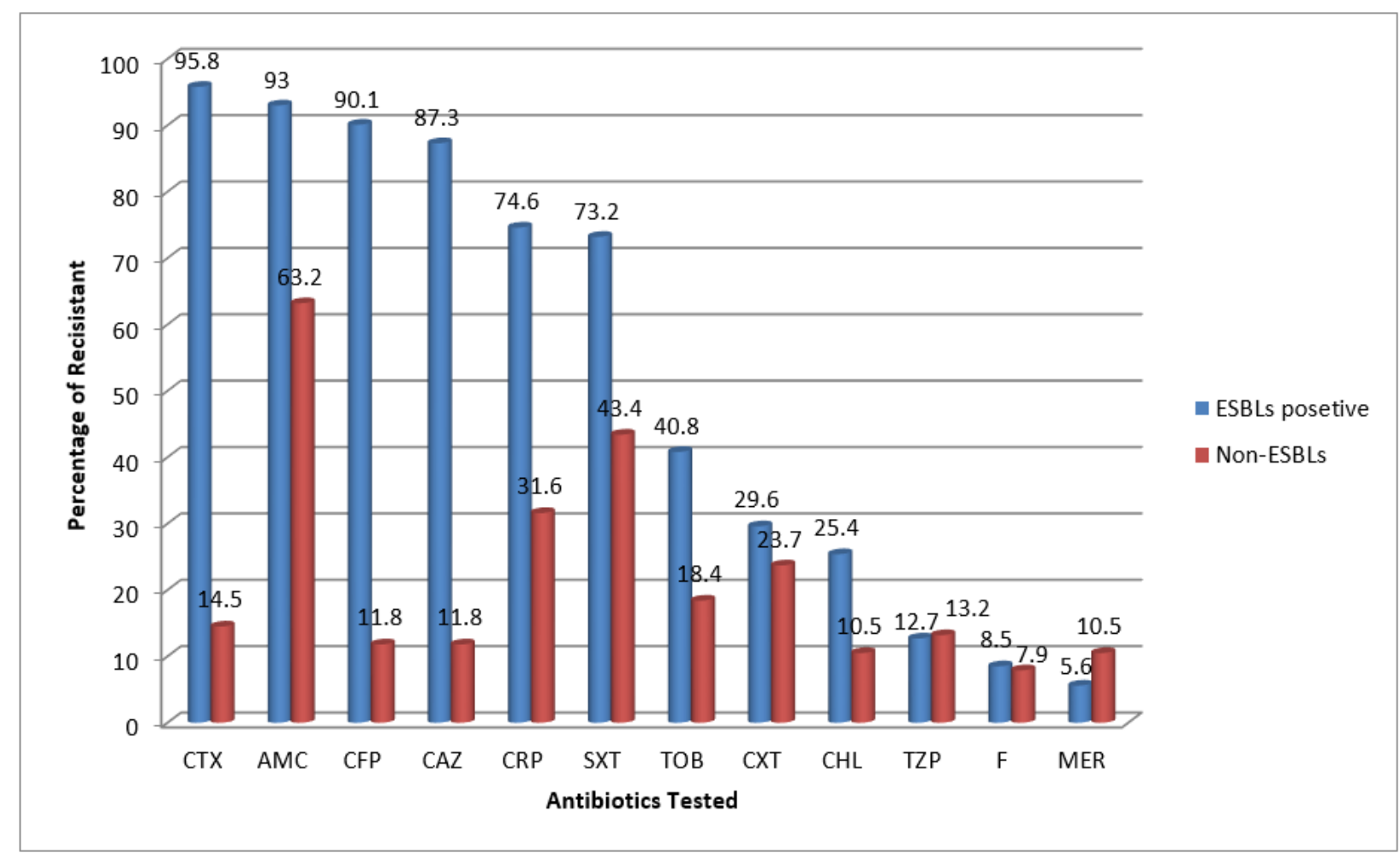

Figure 3

Antibiotics Non-susceptible pattern of ESBLs positive and Non-ESBLs Enterobacteriaceae to different classes of antibiotics at selected hospitals, Addis Ababa, Ethiopia April 1 to May 31, 2020. Note: SXT = Sulfamethoxazole-trimethoprim, CRP= Ciprofloxacin, TZP= Tazobactam/Piperacillin, CXT= Cefoxitin, CHL= Chloramphenicol, $\mathrm{F}=$ nitrofurantoin, $\mathrm{AMC}=$ Amoxicillin-Calvulanic acid, $\mathrm{TOB}=$ Tobramycine, MER= Meropenem, CTX=Cefotaxime, $\mathrm{CFP}=\mathrm{Cefepime,} \mathrm{CAZ}=$ Ceftazidime. 